\title{
redMaGiC: selecting luminous red galaxies from the DES Science Verification data
}
E. Rozo, ${ }^{1 \star}$ E. S. Rykoff,,${ }^{2,3 \star}$ A. Abate,${ }^{1}$ C. Bonnett, ${ }^{4}$ M. Crocce,${ }^{5}$ C. Davis,${ }^{2,6,7}$
B. Hoyle, ${ }^{8}$ B. Leistedt, ${ }^{9}$ H. V. Peiris, ${ }^{9}$ R. H. Wechsler,${ }^{2,3,10}$ T. Abbott, ${ }^{11}$
F. B. Abdalla, ${ }^{9}$ M. Banerji ${ }^{12,13}$ A. H. Bauer, ${ }^{5}$ A. Benoit-Lévy, ${ }^{9}$ G. M. Bernstein, ${ }^{14}$
E. Bertin, ${ }^{15,16}$ D. Brooks, ${ }^{9}$ E. Buckley-Geer, ${ }^{17}$ D. L. Burke,,${ }^{2,3}$ D. Capozzi, ${ }^{18}$

A. Carnero Rosell, ${ }^{19,20}$ D. Carollo, ${ }^{21}$ M. Carrasco Kind, ${ }^{22,23}$ J. Carretero,,${ }^{4,5}$
F. J. Castander, ${ }^{5}$ M. J. Childress, ${ }^{24}$ C. E. Cunha, ${ }^{2}$ C. B. D' Andrea, ${ }^{18}$ T. Davis, ${ }^{2,6,7}$
D. L. DePoy,${ }^{25}$ S. Desai, ${ }^{26,27}$ H. T. Diehl, ${ }^{17}$ J. P. Dietrich, ${ }^{8,26}$ P. Doel,,${ }^{9}$ T. F. Eifler, ${ }^{14,28}$

A. E. Evrard, ${ }^{29,30}$ A. Fausti Neto, ${ }^{19}$ B. Flaugher, ${ }^{17}$ P. Fosalba, ${ }^{5}$ J. Frieman, ${ }^{17,31}$

E. Gaztanaga, ${ }^{5}$ D. W. Gerdes,${ }^{30}$ K. Glazebrook, ${ }^{32}$ D. Gruen, ${ }^{8,33}$ R. A. Gruendl, ${ }^{22,23}$

K. Honscheid, ${ }^{34,35}$ D. J. James, ${ }^{11}$ M. Jarvis,${ }^{14}$ A. G. Kim, ${ }^{36}$ K. Kuehn, ${ }^{37}$

N. Kuropatkin, ${ }^{17}$ O. Lahav, ${ }^{9}$ C. Lidman, ${ }^{6,37}$ M. Lima, ${ }^{19,38}$ M. A. G. Maia, ${ }^{19,20}$

M. March, ${ }^{14}$ P. Martini, ${ }^{34,39}$ P. Melchior, ${ }^{34,35}$ C. J. Miller, ${ }^{29,30}$ R. Miquel, $, 4,40$

J. J. Mohr, ${ }^{26,27,33}$ R. C. Nichol, ${ }^{18}$ B. Nord, ${ }^{17}$ C. R. O’Neill, ${ }^{7}$ R. Ogando, ${ }^{19,20}$
A. A. Plazas, ${ }^{28}$ A. K. Romer, ${ }^{41}$ A. Roodman, ${ }^{2,3}$ M. Sako, ${ }^{14}$ E. Sanchez, ${ }^{42}$
B. Santiago, ${ }^{19,43}$ M. Schubnell, ${ }^{30}$ I. Sevilla-Noarbe, ${ }^{22,42}$ R. C. Smith, ${ }^{11}$
M. Soares-Santos,${ }^{17}$ F. Sobreira, ${ }^{17,19}$ E. Suchyta, ${ }^{34,35}$ M. E. C. Swanson, ${ }^{23}$ J. Thaler, ${ }^{44}$
D. Thomas, ${ }^{18}$ S. Uddin,,${ }^{6,32}$ V. Vikram, ${ }^{45}$ A. R. Walker, ${ }^{11}$ W. Wester, ${ }^{17}$ Y. Zhang ${ }^{30}$ and L. N. da Costa ${ }^{19,20}$

Affiliations are listed at the end of the paper

Accepted 2016 May 25. Received 2016 May 22; in original form 2015 September 16

\begin{abstract}
We introduce redMaGiC, an automated algorithm for selecting luminous red galaxies (LRGs). The algorithm was specifically developed to minimize photometric redshift uncertainties in photometric large-scale structure studies. redMaGiC achieves this by self-training the colour cuts necessary to produce a luminosity-thresholded LRG sample of constant comoving density. We demonstrate that redMaGiC photo-zs are very nearly as accurate as the best machine learning-based methods, yet they require minimal spectroscopic training, do not suffer from extrapolation biases, and are very nearly Gaussian. We apply our algorithm to Dark Energy Survey (DES) Science Verification (SV) data to produce a redMaGiC catalogue sampling the redshift range $z \in[0.2,0.8]$. Our fiducial sample has a comoving space density of $10^{-3}\left(h^{-1} \mathrm{Mpc}\right)^{-3}$, and a median photo- $z$ bias $\left(z_{\text {spec }}-z_{\text {photo }}\right)$ and scatter $\left(\sigma_{z} /(1+z)\right)$ of 0.005 and 0.017 , respectively. The corresponding $5 \sigma$ outlier fraction is 1.4 per cent. We also test our algorithm with Sloan Digital Sky Survey Data Release 8 and Stripe 82 data, and discuss how spectroscopic training can be used to control photo- $z$ biases at the 0.1 per cent level.
\end{abstract}

Key words: methods: statistical-techniques: photometric-galaxies: general. 


\section{INTRODUCTION}

Since the beginning of the Sloan Digital Sky Survey (SDSS; York et al. 2000), it has been recognized that luminous red galaxies (LRGs) are an ideal probe of large-scale structure (LSS; Stoughton et al. 2002). Being luminous, they can be observed to high redshift with relatively shallow exposures. In addition, the $4000 \AA$ break in the spectra of these galaxies enables robust photometric redshift estimates (photo- $z \mathrm{~s}$ ) when the break is photometrically sampled. To date, red galaxy selection algorithms have been fairly crude: one typically defines a colour box that isolates LRGs in colourcolour space, with the specific cuts being selected in a relatively ad hoc manner (e.g. Eisenstein et al. 2001, 2005). This relative lack of attention is driven by the fact that spectroscopic follow-up renders high-precision selection of LRGs unnecessary. With the advent of photometric surveys with no spectroscopic component like the DES (The Dark Energy Survey Collaboration 2005) and the Large Synoptic Survey Telescope (LSST; LSST Science Collaboration. 2009), it is now important to develop selection algorithms designed to minimize photometric redshift uncertainties.

To this end, we have developed redMaGiC, a new red galaxy selection algorithm. Specifically, our primary motivation is to select galaxies with robust, exquisitely controlled photometric redshifts. A secondary and complementary goal is to develop a new photometric redshift estimator for these galaxies that is well understood, and has spectroscopic requirements that are either easily met with existing facilities. The algorithm relies heavily on the infrastructure built for red-sequence cluster finding with redMaPPer (Rykoff et al. 2014, henceforth RM1). Specifically, redMaPPer combines sparse spectroscopy of galaxy clusters with photometric data to calibrate the red sequence of galaxies as a function of redshift. We use the resulting calibration as a photometric template, and select a galaxy as red if this empirical template provides a good description of the galaxy's colour. We refer to the resulting galaxy catalogue as the red-sequence Matched-filter Galaxy Catalog or redMaGiC for short.

We implement our algorithm in the DES Science Verification (SV) data (Rykoff et al. 2016) and characterize the photo- $z$ properties of the resulting catalogue. To provide further photo- $z$ testing, we have also applied redMaGiC to SDSS Data Release 8 (DR8) and SDSS Stripe 82 (S82) data.

The layout of the paper is as follows. Section 2 briefly summarizes the data sets used in this work. Section 3 describes the redMaGiC selection algorithm and the redMaGiC photo- $z$ estimator. Section 4 evaluates the performance of redMaGiC in each of the three data sets considered in this work, while Section 5 compares the red$\mathrm{MaGiC}$ photo- $z$ performance to several other photo- $z$ methods. Section 6 demonstrates that redMaGiC succeeds at selecting galaxies with clean photo- $z$ s by comparing redMaGiC galaxies to the SDSS 'constant mass' CMASS sample, which was specifically tailored for spectroscopic follow-up of galaxies at $z \geq 0.45$ (Dawson et al. 2013). Section 7 discusses how redMaGiC can be improved upon if representative spectroscopic subsamples of redMaGiC galaxies become available. Section 8 characterizes redMaGiC catastrophic failures, which we take to mean $5 \sigma$ outliers. A discussion and summary of our conclusions are presented in Section 9.

Fiducial cosmology and conventions. The construction of the redMaGiC galaxy samples requires that one specify a cosmology for computing the comoving density of galaxies, and for estimating luminosity distances. To do this, we assume a flat $\Lambda$ cold dark matter cosmology with $\Omega_{\mathrm{m}}=0.3$ and $h=1.0$ (i.e. distances are in $\left.h^{-1} \mathrm{Mpc}\right)$. This is the convention used by redMaPPer.
Finally, this work references both $z$-band magnitudes and galaxy redshifts. To avoid confusion, we denote $z$-band magnitudes via $m_{z}$, and reserve the symbol $z$ to signify redshift. Similarly, we refer to $i$-band magnitudes via $m_{i}$ to distinguish from the counting index $i$.

\section{DATA}

\subsection{DES SV data}

DES is a wide-field photometric survey in the griz $Y$ bands performed with the Dark Energy Camera (DECam; Diehl et al. 2012; Flaugher et al. 2015). The DECam is installed at the prime focus of the 4 metre Blanco Telescope at Cerro Tololo Inter-American Observatory (CTIO). The full DES survey is scheduled for 525 nights distributed over five years, covering $5000 \mathrm{deg}^{2}$ of the southern sky, approximately half of which overlaps the South Pole Telescope (SPT; Carlstrom et al. 2011) Sunyaev-Zel'dovich cluster survey.

Prior to the commencement of regular survey operations in 2013 August, from 2012 November to 2013 March DES conducted a $\sim 300 \mathrm{deg}^{2}$ SV survey. The main portion of the SV footprint, used in this paper, covers the $\sim 150 \mathrm{deg}^{2}$ Eastern SPT ('SPTE') region, in the range $65<\mathrm{RA}<93$ and $-60<$ Dec. $<-42$. SPTE was observed between 2 and 10 tilings in each of the griz filters. In addition, DES surveys 10 supernova (SN) fields every 5-7 d, each of which covers a single DECam 2.2-wide field of view. The median depth of the SV survey (defined as $10 \sigma$ detections for extended sources) is $g=24.0, r=23.9 i=23.0, z=22.3$, and $Y=20.8$.

The DES SV data were processed by the DES Data Management infrastructure (Gruendl et al., in preparation). This processing performs image deblending, astrometric registration, global calibration, image co-addition, and object catalogue creation. Details of the DES single-epoch and co-add processing can be found in Sevilla et al. (2011) and Desai et al. (2012). We use SExtractor to create object catalogues from the single-epoch and co-added images (Bertin \& Arnouts 1996; Bertin 2011). Object detection was performed on a 'chi-squared' co-add of the $r+i+z$ image with SWARP (Bertin 2010), and object measurement was performed in dual-image mode with each individual griz image (here we ignore the shallow $Y$-band imaging).

After production of these early data, several problems were detected and corrected for in post-processing, leading to the creation of the 'SVA1 Gold' catalogue (Rykoff et al. 2016). First, unmasked satellite trails were masked. Secondly, calibration was improved using a modified version of the BIG-MACs stellar-locus fitting code (Kelly et al. 2014). ${ }^{1}$ We recomputed co-add zero-points over the full SV footprint on a HEALPix (Górski et al. 2005) grid of NSIDE $=256$. These zero-points were then interpolated with a bilinear scheme to correct the magnitudes of all objects in the catalogue. Finally, regions around bright stars $(J<13)$ from the Two Micron All Sky Survey (Skrutskie et al. 2006) were masked.

Galaxy magnitudes and colours are computed via the SEXTRACTOR MAG_AUTO quantity. These colours are significantly noisier than those obtained through model fitting. However, for SV co-added images, MAG_AUTO colours are considerably more stable due to point spread function (PSF) discontinuities in the co-added images sourced by co-adding different exposures. This is expected to have a negative impact on our results, and future work will make use of full galaxy multi-epoch multi-band colour measurements.

\footnotetext{
${ }^{1}$ https://code.google.com/p/big-macs-calibrate/
} 


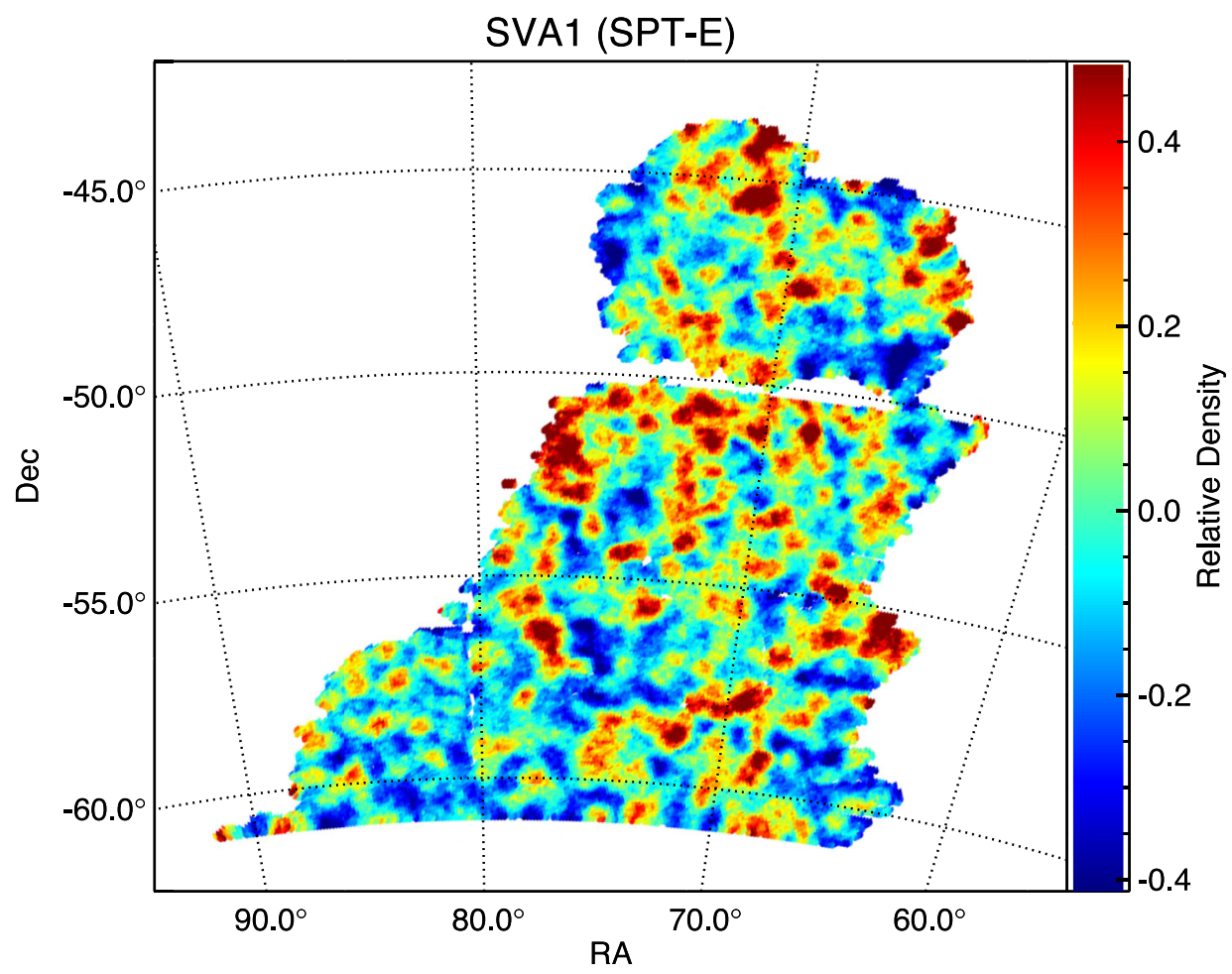

Figure 1. Angular galaxy density contrast $\delta=(\rho-\bar{\rho}) / \bar{\rho}$ for DES SV redMaGiC galaxies in the redshift range [0.2, 0.8], averaged on a 15 arcmin scale. This plot uses our fiducial redMaGiC sample (see the text).

Star-galaxy separation is a particularly challenging issue for red galaxy selection at high redshift. In particular, at $z \sim 0.7$ the red end of the stellar locus approaches the red-sequence galaxy locus when using purely optical ( $g$ riz) photometry. Therefore, we have made use of the NGMIX multi-band multi-epoch image processing (Jarvis et al. 2016; Sheldon et al., in preparation) to select a relatively pure and complete galaxy selection. Details are presented in Appendix A. As NGMIX is primarily used for shape measurements on DES data, the tolerance for input image quality is relatively tight, so our footprint is smaller than that of SVA1 Gold (see Jarvis et al. 2016). Finally, we only consider regions where the $z$-band $10 \sigma$ depth in MAG_AUTO has $m_{z}>22$ (Rykoff et al. 2016). In total, we use $148 \mathrm{deg}^{2}$ of DES $\mathrm{SV}$ imaging in this paper, and the angular mask is described in Appendix B.

We note that redMaGiC relies on the red-sequence calibration by the redMaPPer algorithm, as detailed in RM1. The DES SV redMaPPer cluster catalogue is described in Rykoff et al. (2016). We refer the reader to that work for a detailed description of the catalogue. Here, we simply note that the redMaPPer calibration of the red sequence requires spectroscopic training data for galaxy clusters. This spectroscopic data set is primarily comprised of existing external spectroscopic surveys, including the Galaxy and Mass Assembly survey (GAMA; Driver et al. 2011), the VIMOS VLT Deep Survey (Garilli et al. 2008), the 2dF Galaxy Redshift Survey (Colless et al. 2001), SDSS (Ahn et al. 2014), the VIMOS Public Extragalactic Survey (VIPERS; Garilli et al. 2014), the UKIDSS Ultra-Deep Survey (Bradshaw et al. 2013; McLure et al. 2013), and the Arizona CDFS Environment Survey (Cooper et al. 2012). In addition, we have a small sample of cluster redshifts from SPT used in the cluster validation (Bleem et al. 2015). These data sets have been further supplemented by galaxy spectra acquired as part of the
OzDES spectroscopic survey, which is performing spectroscopic follow-up on the AAOmega instrument at the Anglo-Australian Telescope in the DES SN fields (Yuan et al. 2015). The total number of spectroscopic cluster redshifts used in our calibration is 625 , most of which are low richness. By point of comparison, current DES machine learning methods rely on over 46000 spectra.

Fig. 1 shows the angular density contrast of our fiducial redMaGiC galaxy sample in the so-called DES SV SPTE region. The full DES SV catalogue also includes the DES SN fields, which are disconnected from the SPTE field. We note that very nearly all the spectroscopic training data sets reside in the DES SN field, which places significant limitations in our ability to validate the performance of redMaGiC on the DES SV data set.

We note that the survey depth varies significantly over the footprint. In some regions, we can comfortably reach high redshifts $(z \lesssim 1)$, while in other regions the depth is insufficient. To obtain a homogeneous catalogue across the full footprint, we restrict ourselves to redMaGiC galaxies over the redshift range $z \in[0.2$, $0.8]$.

\subsection{SDSS DR8 data}

We apply the redMaGiC algorithm to SDSS DR8 photometric data (Aihara et al. 2011). The DR8 galaxy catalogue contains $\approx 14000 \mathrm{deg}^{2}$ of imaging, which we reduce to $\approx 10000 \mathrm{deg}^{2}$ of contiguous high-quality observations using the mask from the Baryon Oscillation Spectroscopic Survey (BOSS; Dawson et al. 2013). The mask is further extended to include all stars in the Yale Bright Star Catalog (Hoffleit \& Jaschek 1991), as well as the area around objects in the New General Catalog (Sinnott 1988). The resulting mask is that used by RM1 to generate the SDSS DR8 redMaPPer 
catalogue. We refer the reader to that work for further discussion on the mask.

Galaxies are selected using the default SDSS star/galaxy separator. We filter all galaxies with any of the following flags in the $g, r$, or $i$ band: SATUR CENTER, BRIGHT, TOO MANY PEAKS, and (NOT BLENDED OR NODEBLEND). Unlike the BOSS target selection, we keep objects flagged with SATURATED, NOTCHECKED, and PEAKCENTER. A discussion of these choices can be found in RM1. Total magnitudes are determined from $i$-band CMODEL_MAG and colours from ugriz MODEL_MAG.

The red-sequence model is that of the SDSS DR8 redMaPPer v6.3 cluster catalogue (Rykoff et al. 2016). This catalogue is an updated version of the redMaPPer catalogue in RM1 (v5.2), and supersedes both it and the update in Rozo et al. (2015, v5.10). Spectroscopic training data are drawn from the SDSS DR10 spectroscopic data set (Ahn et al. 2014).

\subsection{SDSS S82 data}

We apply the redMaGiC algorithm on SDSS S82 co-add data (Annis et al. 2014). The $S 82$ catalogue consists of $275 \mathrm{deg}^{2}$ of ugriz coadded imaging over the equatorial stripe. The co-add is roughly 2 mag deeper than the single-pass SDSS data. We use the same flag cuts as those used for the DR8 catalogue. In addition, we clean all galaxies with extremely large magnitude errors. Total magnitudes are determined from $i$-band CMODEL_MAG and colours from griz MODEL_MAG. Most modest- to high-redshift $(z \gtrsim 0.3)$ red galaxies in S82 are $u$-band dropouts, so we opted to rely exclusively on griz photometry for S82 runs. However, in Section 8, we demonstrate the utility of the $u$-band imaging at low redshift.

We have run the redMaPPer algorithm in this photometric data set, using SDSS DR10 spectroscopy as the spectroscopic training data set. In addition, for high-redshift performance validation, we make use of VIPERS (Franzetti et al. 2014). During our testing and validation of the redMaPPer catalogue on these data, we discovered that $\approx 15$ per cent of red cluster member galaxies in the $S 82$ data set have reported magnitudes that are clearly incorrect in one or more bands. We do not know the origin of this failure, nor whether it extends to other galaxies (blue cluster galaxies or field galaxies). These errors inevitably bias the resulting cluster richness estimates. Consequently, we have opted not to release the S82 redMaPPer and redMaGiC catalogues. Nevertheless, we include a discussion of these data because the photo- $z$ performance of redMaGiC in this data set provides a valuable baseline to compare against the DES $\mathrm{SV}$ redMaGiC sample.

\section{THE redMaGiC SELECTION ALGORITHM}

The redMaGiC algorithm can be summarized very simply.

(i) Fit every galaxy to a red-sequence template. Compute the corresponding best-fitting redshift $z_{\text {photo }}$, and the goodness-of-fit $\chi^{2}$ of the template fit.

(ii) Given $z_{\text {photo }}$, compute the galaxy luminosity $L$.

(iii) If the galaxy is bright ( $\left.L \geq L_{\min }\right)$, and it is a good fit to the red-sequence template $\left(\chi^{2} \leq \chi_{\max }^{2}\right)$, include it in the redMaGiC catalogue. Otherwise, drop it.

As long as $\chi_{\max }^{2}$ is sufficiently aggressive, the resulting catalogue will be very nearly comprised of red-sequence galaxies exclusively. In addition, if the red-sequence photometric template is accurate, then the resulting redshifts should be of excellent quality. In what follows, we describe how we construct our red-sequence template, and how the maximum goodness-of-fit value $\chi_{\max }^{2}$ is selected so as to ensure that the resulting redMaGiC galaxy sample has a constant comoving space density. It should be noted that our template is not a spectroscopic template. Rather, we model the colours as a function redshift and magnitude directly, without ever going through a spectrum. When we refer to redMaGiC template, we always mean our model colours.

\subsection{The redMaGiC template}

The redMaGiC algorithm relies on the redMaPPer calibration of the red sequence, so we begin our discussion by reviewing how the redMaPPer template is constructed. Let $c$ be the colour vector of a galaxy and $m$ denote the galaxy's magnitude in some reference band. When possible, the reference band should lie redwards of the $4000 \AA$ break at all redshifts, which leads us to select $m_{z}$ as the reference magnitude for the DES redMaGiC sample. The lower redshift range of the SDSS catalogues allows us to use $m_{i}$ in those data sets. One could in principle use $m_{z}$ in SDSS as well, but since SDSS $m_{i}$ is much less noisy than $m_{z}$, we rely on the $i$ band for the SDSS data.

Red-sequence galaxies populate a narrow ridgeline in colourmagnitude space, though with some intrinsic scatter, which we model as Gaussian. In this case, the ridgeline corresponds to the mean colour of red-sequence galaxies. We write

$\langle\boldsymbol{c} \mid m, z\rangle=\boldsymbol{a}(z)+\boldsymbol{\alpha}(z)\left(m-m_{\mathrm{ref}}(z)\right)$.

Here $\boldsymbol{a}(z)$ and $\boldsymbol{\alpha}(z)$ are the unknown redshift-dependent amplitude and slope of the red sequence. The magnitude $m_{\text {ref }}(z)$ defines the pivot point of the colour-magnitude relation. Its value is arbitrary and can be freely chosen by the experimenter. redMaPPer selects $m_{\text {ref }}(z)$ so that it traces the median magnitude of the cluster member galaxies. The unknown functions $\boldsymbol{a}(z)$ and $\boldsymbol{\alpha}(z)$ are parametrized via spline interpolation, with the model parameters being the value of the functions at a grid of redshifts.

The covariance matrix $\mathbf{C}_{\text {int }}$ characterizing the intrinsic width of the red sequence in multi-dimensional colour space is assumed to be independent of magnitude. The covariance matrix is, however, assumed to vary as a function of redshift. As with the functions $\boldsymbol{a}(z)$ and $\boldsymbol{\alpha}(z)$, the matrix $\mathbf{C}_{\mathrm{int}}(z)$ is parametrized via spline interpolation, with the model parameters being the values of each independent matrix element along a grid of redshifts. Together with the parameters for $\boldsymbol{a}(z)$ and $\boldsymbol{\alpha}(z)$, this set of model parameters $\boldsymbol{p}$ fully specifies the colour distribution of red-sequence galaxies $P(\boldsymbol{c} \mid \boldsymbol{p} ; m, z)$.

The parameters $\boldsymbol{p}$ specifying our colour model are fitted using an iterative maximum likelihood approach. Briefly, given a cluster galaxy with a spectroscopic redshift $z_{\text {spec }}$, and a rough estimate for the parameters $\boldsymbol{p}$, one can photometrically select cluster galaxies using a matched-filter approach. Given these initial photometric cluster members, one then defines the likelihood

$\mathcal{L}(\boldsymbol{p})=\prod P\left(\boldsymbol{c}_{i} \mid \boldsymbol{p} ; m_{i}, z_{\text {cluster }}\right)$,

where the product is over all the selected cluster members. In practice, the likelihood is modified to allow for contamination by interlopers (RM1). A new set of parameters $\boldsymbol{p}$ is estimated by maximizing the above likelihood, and the whole procedure is iterated until convergence. For further details, see RM1. The end result of the above procedure is a strictly empirical calibration of the red sequence of cluster galaxies as a function of redshift. 


\section{2 redMaGiC photometric redshifts}

We want to estimate the photometric redshift of a galaxy of magnitude $m$ and colour $\boldsymbol{c}$. We use an updated version of the photometric redshift estimator $z_{\text {red }}$ introduced in RM1. The probability that a red galaxy selected from a constant comoving density sample has redshift $z$, magnitude $m$, and colour $c$ is denoted via $P(c, m, z)$. One has

$P(\boldsymbol{c}, m, z)=P(\boldsymbol{c} \mid m, z) P(m \mid z) P(z)$.

We are interested in the redshift probability distribution

$$
\begin{aligned}
P(z \mid \boldsymbol{c}, m) & =\frac{P(\boldsymbol{c}, m, z)}{P(\boldsymbol{c}, m)} \\
& =\frac{P(\boldsymbol{c} \mid m, z) P(m \mid z) P(z)}{P(\boldsymbol{c}, m)} .
\end{aligned}
$$

Since the denominator is redshift independent, we can ignore it. The corresponding likelihood is

$$
\mathcal{L}(z)=P(\boldsymbol{c} \mid m, z) P(m \mid z) P(z) .
$$

For a constant comoving density sample $P(z) \propto|\mathrm{d} V / \mathrm{d} z| . P(m \mid z)$ is modelled assuming that the galaxies follow a Schechter luminosity function,

$$
P(m \mid z) \propto 10^{-0.4\left(m-m_{*}\right)(\alpha+1)} \exp \left[-10^{-0.4\left(m-m_{*}\right)}\right] .
$$

The value $m_{*}(z)$ is set to $m_{i}=17.85$ at $z=0.2$ to match redMaPPer. The evolution of $m_{*}(z)$ is computed using the Bruzual \& Charlot (2003, BC03) stellar population synthesis code as implemented in the EZGAL PYTHON package. ${ }^{2}$ We model $m_{*}(z)$ using a single star formation burst at $z=3$, and we have confirmed that this evolution matches that in RM1 at $z<0.5$. The normalization condition for $m_{z}$ for DES is then derived from the BC03 model using the DECam passband. Finally, $P(\boldsymbol{c} \mid m, z)$ of our red-sequence model is

$P(\boldsymbol{c} \mid m, z) \propto \exp \left(-\frac{1}{2} \chi^{2}(z)\right)$

where

$\chi^{2}(z)=(\boldsymbol{c}-\langle\boldsymbol{c} \mid m, z\rangle) \mathbf{C}_{\mathrm{tot}}^{-1}(\boldsymbol{c}-\langle\boldsymbol{c} \mid m, z\rangle)$

and

$\mathbf{C}_{\text {tot }}=\mathbf{C}_{\text {int }}+\mathbf{C}_{\text {obs }}$

is the total scatter about the red-sequence colour. Here, $\mathbf{C}_{\mathrm{obs}}$ is the covariance matrix describing the photometric errors in the galaxy colours. Our final expression for the redshift likelihood is therefore

$$
\ln \mathcal{L}(z)=-\frac{1}{2} \chi^{2}(z)+\ln P(m \mid z)+\ln \left|\frac{\mathrm{d} V}{\mathrm{~d} z}\right| .
$$

The photometric redshift $z_{\text {red }}$ is the redshift at which this loglikelihood function is maximized, and the corresponding $\chi^{2}$ value is denoted $\chi_{\text {red }}^{2}$. In addition, the galaxy is also assigned a luminosity $l=L / L_{*}\left(z_{\text {red }}\right)$,

$l\left(m, z_{\text {red }}\right)=\frac{L}{L_{*}}=10^{-0.4\left(m-m_{*}\left(z_{\text {red }}\right)\right)}$.

The photometric redshift error $\sigma_{z}$ is estimated using the variance of the posterior,

$\sigma_{z}^{2}=\left\langle z^{2}\right\rangle-\langle z\rangle^{2}$

\footnotetext{
${ }^{2}$ http://www.baryons.org/ezgal
}

where

$\left\langle z^{n}\right\rangle=\frac{\int \mathrm{d} z \mathcal{L}(z) z^{n}}{\int \mathrm{d} z \mathcal{L}(z)}$.

\subsection{Selection cuts}

We wish to select LRGs. Consequently, we demand that all galaxies have a luminosity $l \geq l_{\min }$, where $l_{\min }=L_{\min } / L_{*}$ is a selection parameter that is to be determined by the experimenter. To ensure that our final galaxy sample is comprised of red-sequence galaxies, we further demand that our red-sequence template be a good fit by applying the selection cut

$\chi_{\text {red }}^{2} \leq \chi_{\max }^{2}\left(z_{\text {red }}\right)$.

Note that the $\chi^{2}$ cut $\chi_{\max }^{2}(z)$ can be redshift dependent. The simplest possible model is $\chi_{\max }^{2}(z)=k$ for some constant $k$, but this is rather arbitrary. What we really want is to be able to select the 'same' sample of galaxies at all redshifts. In the absence of merging, red-sequence galaxies evolve passively, resulting in a constant comoving density sample. Of course, galaxies do merge, so this approximation cannot be exactly correct, but this can nevertheless be a useful approximation for comparing galaxies across relatively narrow redshift intervals. Thus, rather than applying a constant $\chi^{2}$ cut, we construct the selection threshold $\chi_{\max }^{2}(z)$ such that the resulting galaxy sample has a constant comoving galaxy density. This selection also justifies our assumption that $P(z) \propto|\mathrm{d} V / \mathrm{d} z|$ in the construction of the redshift likelihood.

To ensure a constant comoving space density of redMaGiC galaxies, we parametrize $\chi_{\max }^{2}(z)$ using spline parametrization. The model parameters $\boldsymbol{q}$ are the values of $\chi_{\max }^{2}$ along a grid of redshifts, and the value of $\chi_{\max }^{2}(z)$ everywhere else is defined via spline interpolation. We will come back to how the parameters $\boldsymbol{q}$ are chosen momentarily. Before we do so, however, we need to describe an additional calibration step we take in order to improve the photometric redshift performance of the redMaGiC algorithm.

\subsection{Photo- $z$ afterburner}

The redMaGiC selection cuts are fully specified by the parameter $l_{\min }$ and the parameters $\boldsymbol{q}$ defining the function $\chi_{\max }^{2}(z)$. If a random fraction of the selected galaxies have spectroscopic redshifts $z_{\text {spec }}$, we can use these galaxies to remove any biases in our photo- $z$ s. For instance, given the redMaGiC selection specified by $l_{\min }$ and $\boldsymbol{q}$, we could split the spectroscopic galaxies into two, a training sample and a validation sample. We can then use the training sample to compute the median redshift offset $z_{\text {spec }}-z_{\text {red }}$ in bins of $z_{\text {red }}$. We denote this quantity as $\Delta z\left(z_{\text {red }}\right)$. Our new photometric redshift estimator is

$z_{\mathrm{rm}}=z_{\mathrm{red}}+\Delta z\left(z_{\mathrm{red}}\right)$

which we can validate with the validation data set.

In practice, $\Delta z\left(z_{\text {red }}\right)$ is defined using spline interpolation, with the spline parameters being determined by minimizing the cost function

$E_{\Delta}=\sum_{j}\left|z_{\text {spec }, j}-z_{\mathrm{rm}, j}\right|$,

where the sum is over all spectroscopic redMaGiC galaxies. We add the absolute values rather than the squares to reduce the impact of possible catastrophic outliers.

Of course, in general one is hardly assured of spectroscopic redshifts for a large representative sample of redMaGiC 
galaxies. We overcome this problem by relying instead on red$\mathrm{MaGiC}$ galaxies that are members of redMaPPer clusters (membership probability $p_{\text {mem }} \geq 0.9$ ), using the redMaPPer photometric cluster redshift $z_{\lambda}$ as the 'spectroscopic' redshift of the calibration galaxies. Roughly, the redshift $z_{\lambda}$ is obtained by simultaneously fitting the ensemble of cluster galaxies with a single photometric redshift. It has already been shown that redMaPPer redshifts are unbiased and much more accurate than the photometric redshifts of individual galaxies. We emphasize that by making use of photometric cluster members, our calibration sample is not restricted to the brightest redMaGiC galaxies, as would be the case of a typical spectroscopic calibration sample.

In addition to modifying the photometric redshift estimate $z_{\mathrm{rm}}$, we also modify the photometric redshift errors. Imagine again binning the galaxy calibration sample by $z_{\mathrm{rm}}$. For each bin, we could compute the median absolute deviation, $\operatorname{MAD}=\operatorname{median}\left\{\mid z_{\text {red }}-\right.$ $\left.z_{\lambda} \mid\right\}$. For a Gaussian distribution, $\langle\mathrm{MAD}\rangle=\sigma_{z} / 1.4826$, where $\sigma_{z}$ is the standard deviation. Thus, the quantity $1.4826\left|z_{\mathrm{rm}}-z_{\lambda}\right|$ is an estimator for $\sigma_{z}$. Let then $\sigma_{0}$ be our original photometric redshift error estimate as per Section 3.2. We assume that the corrected photometric redshift error $\sigma_{1}$ for each galaxy is given by $\sigma_{1}=$ $r\left(z_{\mathrm{rm}}\right) \sigma_{0}$, where $r\left(z_{\mathrm{rm}}\right)=\sigma_{z} / \sigma_{0}$. Rather than doing this in bins, we parametrize $r(z)$ via spline interpolation, with the best-fitting parameters being those which minimize the cost function

$E_{\sigma}=\sum_{j}|1.4826| z_{\mathrm{rm}, j}-z_{\lambda, j}\left|-r\left(z_{\mathrm{rm}, j}\right) \sigma_{0, j}\right|$.

The sum is over all calibration galaxies, and we again use absolute values to reduce the impact of possible catastrophic outliers. We note that the afterburner perturbations to the photometric redshifts are small, but do improve photometric redshift performance.

With the new estimator $z_{\mathrm{rm}}$ in hand and its improved error estimate, we can recompute the luminosity $l$ and $\chi^{2}$ of every galaxy in the survey, and reapply our selection cuts to arrive at an improved redMaGiC sample.

\section{$3.5 \chi_{\max }^{2}$ calibration}

We have seen how to select redMaGiC galaxies given the selection parameters $\boldsymbol{q}$, but we have yet to specify how the parameters $\boldsymbol{q}$ are selected. To do so, we first define a series of redshift bins $z_{j}$ going from the minimum redshift of interest $z_{\min }$ to the maximum redshift $z_{\max }$. Given a set of selection parameters $\boldsymbol{q}$, we construct the redMaGiC sample by applying the luminosity and $\chi^{2}$ cuts as above. Next, we compute the photo- $z$ afterburner parameters for the sample derived from the parameters $\boldsymbol{q}$, which allows us to compute $z_{\mathrm{rm}}$ for every galaxy. We then measure the comoving space density $n_{j}(\boldsymbol{q})$ in each redshift bin $j$. Since we want to enforce a constant comoving density $\bar{n}$, we define the cost function $E(\boldsymbol{q})$ via

$E(\boldsymbol{q})=\sum_{j} \frac{\left(n_{j}(\boldsymbol{q})-\bar{n}\right)^{2}}{\bar{n} V_{j}^{-1}}$,

where the sum is over all redshift bins, $V_{j}$ is the comoving volume of redshift bin $j$, and $n_{j}$ is the empirical redMaGiC galaxy density in redshift bin $j$. The denominator is the expected Poisson error for a galaxy density $\bar{n}$. The spline parameters $\boldsymbol{q}$ are obtained by minimizing the cost function $E(\boldsymbol{q})$ using the downhill-simplex method of Nelder \& Mead (1965). We always use redshift bins that are significantly narrower than the spacing between spline nodes, and we take care to ensure that the number of galaxies $n_{j} V_{j} \gg 1$ in every redshift bin. We emphasize that the photo- $z$ afterburner parameters are re-estimated at every iteration in the minimization, to ensure that we have a consistent sample selection given the updated galaxy redshifts. Finally, with the spline parameters determined, we apply the corresponding $\chi_{\text {red }}^{2} \leq \chi_{\max }^{2}\left(z_{\mathrm{rm}}\right)$ cut to arrive at the final redMaGiC galaxy sample.

\subsection{Selection summary}

Despite the computational complexity of the above selection, it is worth emphasizing that our selection algorithm contains only two free parameters, both of which have clear physical interpretations: the luminosity cut $l_{\min }$ and the desired space density $\bar{n}$ of the resulting galaxy sample. Importantly, the 'colour cuts' that select red galaxies are self-trained from the data. By comparison, the SDSS CMASS galaxy selection involves 12 parameters hand-picked a priori to produce an approximately stellar-mass-limited sample at $z \geq 0.45$ (Dawson et al. 2013).

It is also important to note that our selection makes it very easy to test different selection thresholds, allowing one to optimize galaxy selection for scientific purposes. Some patterns emerge: $l_{\min }$ must always be low enough for the corresponding $\chi_{\max }^{2}$ threshold to be reasonable (i.e. $\chi^{2} /$ dof $\lesssim 2$ ). If $l_{\min }$ is too large, redMaGiC will start pulling in galaxies with large $\chi^{2}$ values in order to attempt to reach the desired space density, which will result in a large number of photo- $z$ outliers. We find that when this happens, it becomes difficult to construct a truly flat $n(z)$ sample, so checking the comoving space density of the redMaGiC catalogue is a quick and easy way to test whether the redMaGiC algorithm is performing as desired.

We illustrate the performance of our algorithm in Fig. 2 for a set of fiducial cuts $l_{\min }=0.5$ and $\bar{n}=10^{-3} \mathrm{~h}^{3} \mathrm{Mpc}^{-3}$. The left-hand panel shows the $\chi^{2}(z)$ threshold for each of our three redMaGiC samples, while the right-hand panel shows the resulting galaxy comoving densities as a function of redshift. We see that in all cases the observed space density is close to flat, and that the $\chi^{2}$ thresholds are low, as desired.

\section{PHOTO- $z$ PERFORMANCE}

We consider two sets of redMaGiC galaxies. The first is our fiducial sample, selected to be galaxies brighter than $0.5 L_{*}$ and with a space density $\bar{n}=10^{-3} h^{3} \mathrm{Mpc}^{-3}$. Unless otherwise stated, all of the results noted below correspond to these fiducial selection parameters. The second sample is a high-luminosity, low-spacedensity redMaGiC sample, comprised of galaxies brighter than $L_{*}$ with a space density of $2 \times 10^{-4} h^{3} \mathrm{Mpc}^{-3}$. This high-luminosity sample will be useful for comparing against other commonly used galaxy samples, particularly CMASS.

Fig. 3 shows the photometric redshift performance for our fiducial selection in the SV, DR8, and S82 data sets. The spectroscopic data used to characterize the photometric redshift performance were described in Section 2. The photometric redshift bias $\overline{\Delta z}$ is defined as the median offset of $\Delta z=z_{\mathrm{spec}}-z_{\mathrm{rm}}$. The scatter is defined as $1.4826 \times$ MAD, where MAD is the median absolute deviation, i.e. the median of $|\Delta z-\overline{\Delta z}|$. For Gaussianly distributed data, $\overline{\Delta z}$ and $1.4826 \times$ MAD are unbiased estimators of the mean and standard deviation of these offsets. In using median statistics, our results are robust to a small fraction of gross outliers.

The most obvious features in the left-hand plots of Fig. 3 are the three clumps of outlier points. These are obvious for both DR8 and S82 data, but not apparent in the DES SV data. We are confident that this reflects the paucity of spectra in the DES data rather than a sudden and unexpected improvement in the redMaGiC performance. We discuss each of these clumps in Section 8. 

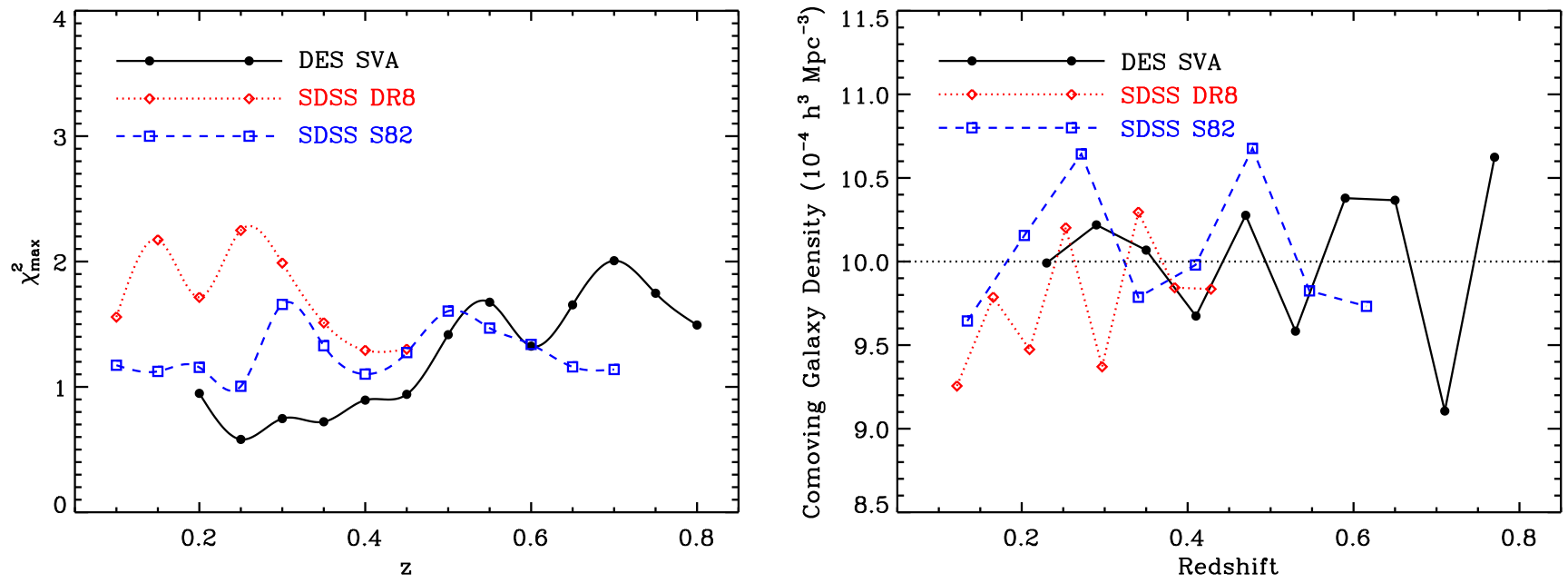

Figure 2. Left: selection cut $\chi_{\max }^{2}$ as a function of redshift defining each of the redMaGiC galaxy samples, as labelled. The symbols mark the spline nodes defining the function $\chi_{\max }^{2}(z)$, while the lines show the corresponding spline interpolation at every point. Right: redMaGiC comoving galaxy density as a function of redshift for each of the three data sets employed in this work, as labelled. The target comoving space density was $10^{-3} h^{3} \mathrm{Mpc}^{-3}$ (horizontal dotted line).

Turning to the bias and scatter plots in the right column of Fig. 3, we see that for all data sets there is excellent agreement between the observed redshift scatter (red solid line) and the predicted photo$z$ uncertainty (dashed blue line). The latter is simply the median photo- $z$ error in each bin. Note that the predicted redshift errors in the SDSS S82 and DES SV data sets are clearly double-humped. This is expected: photometric redshift uncertainties increase whenever the $4000 \AA$ break feature in the spectra of these galaxies falls in between filters. At $z \approx 0.35$, there is a peak associated with the $g$-to$r$ filter transition, and at $z \approx 0.65$ we see a second peak associated with the $r$-to- $i$ filter transition.

Comparing the three data sets, we see DR8 and S82 have nearly identical photometric redshift errors at low redshifts, which demonstrates that the redshift errors are set by the intrinsic width of the red sequence. By contrast, at $z \gtrsim 0.4$ the photometric errors in DR 8 are clearly important, and so its photo- $z$ errors are larger than those in S82. Notably, DES has larger photometric redshift scatter than the SDSS data sets. There are several contributors to this result. First, the spectroscopic training set for redMaPPer training is still quite sparse, and so the redMaPPer calibration is expected to be noisier than in the SDSS data sets. Secondly, DES SV MAG_AUTO colours are expected to be intrinsically noisier than SDSS MODEL_MAG colours, leading to larger uncertainties.

Turning to the bias, we see that the DR8 redMaGiC sample appears to have a negative bias at $z_{\mathrm{rm}} \approx 0.3$. By contrast, the $\mathrm{S} 82$ sample exhibits a slight positive bias at the same photometric redshift. The situation reverses at $z \approx 0.25$, with $\mathrm{S} 82$ galaxies exhibiting bias while DR8 galaxies do not. We believe that these biases are driven by non-representative spectroscopic sampling of redMaGiC galaxies. Specifically, our photometric redshift tests rely on the subset of redMaGiC galaxies that have spectra. If that subset is biased relative to the full population, we would in fact expect to see a photometric redshift bias.

Fig. 4 shows redMaGiC galaxy density contours in the $g-r$ versus $r-i$ plane for several photometric redshift bins. The filled red and orange contours show the regions containing 68 and 95 per cent of all redMaGiC galaxies with spectroscopic redshifts. The solid ellipses show the corresponding regions for all redMaGiC galaxies with a magnitude threshold set by the spectroscopic redMaGiC subsample. Offsets between the red-orange contours and the solid line contours imply a non-representative spectroscopic sampling of the redMaGiC galaxy population.

It is clear from Fig. 4 that DR8 spectroscopic sampling is biased at $z \gtrsim 0.3$, with the reddest galaxies start being somewhat oversampled. There is a similar trend of oversampling the reddest redMaGiC galaxies in $\mathrm{S} 82$ starting at $z \approx 0.23$. These differences appear to be correlated with the presence of 'large' photo- $z$ biases in Fig. 3 .

The photo- $z$ bias at $z \approx 0.6$ in the S82 data is rather unusual. It is large and negative $(\approx-0.005)$ when using SDSS spectroscopy, but large and positive $(\approx 0.009)$ when using VIPERS. The difference between the two spectroscopic data sets further highlights the importance that spectroscopic sampling can have on our conclusions.

The origin of the redshift biases in the DES SV redMaGiC sample is much more difficult to ascertain. First, the spectroscopic training set for redMaPPer is very sparse, and is most certainly not representative of the sample as a whole. For instance, there is a dearth of spectroscopic galaxies at $z \approx 0.4$. A histogram of the number of redMaGiC galaxies as a function of redshift is shown in Fig. 5, along with a contour plot showing how these galaxies populate the redshift-magnitude space. Secondly, most of the redshifts available to us come from training sets in the SN fields, adding up to $\approx 30 \mathrm{deg}^{2}$. The small area results in only a handful of spectroscopic clusters for red-sequence calibration. Thirdly, our reliance on MAG_AUTO colours in the DES is expected to adversely affect photo- $z$ performance. Fortunately, all of these difficulties will be considerably ameliorated if not entirely removed as the DES images larger areas and updates the data reduction pipelines.

A summary of the statistical performance of redMaGiC is presented in Table 1.

\section{COMPARISON TO EXISTING PHOTO- $z$ ALGORITHMS}

\subsection{DR8 comparisons}

As noted in the introduction, redMaGiC seeks both to select galaxies with robust photometric redshifts and to develop a photometric redshift estimator that can be used on these galaxies with minimal 

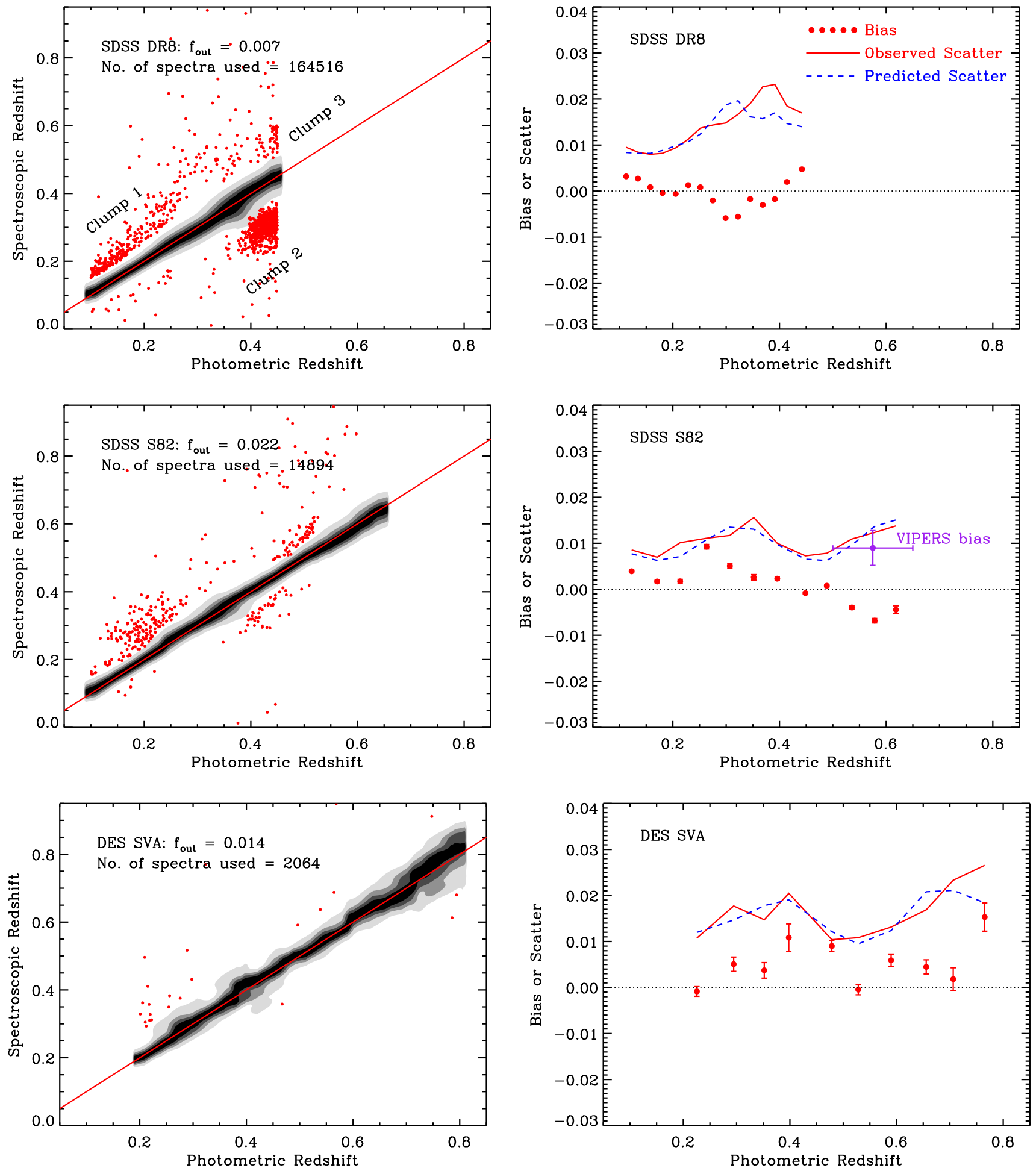

Figure 3. Left: spectroscopic redshift versus photometric redshift for the fiducial redMaGiC galaxy sample in each of the various data sets considered in this work. Red points are $5 \sigma$ outliers, while the red line corresponds to $z_{\mathrm{spec}}=z_{\text {photo }}$. Right: photometric redshift performance statistics. Red points with error bars are the photometric redshift bias, defined as the median value of $z_{\text {spec }}-z_{\text {photo }}$. All statistics for the SDSS data sets are computed using SDSS spectroscopy, except for the purple VIPERS point for S82. The red curve is the observed scatter of $\left(z_{\text {photo }}-z_{\text {spec }}\right) /\left(1+z_{\text {spec }}\right)$, while the dashed blue curve is the predicted scatter based on the available photometry. The horizontal error bar for the S82 plot shows the width of the redshift bin used in the VIPERS measurement.

spectroscopic training data. For the latter to be useful, however, the performance of our algorithm must be comparable to that of existing algorithms. We now test how the redMaGiC photo-zs compare with state-of-the-art photometric redshift codes run on redMaGiC galaxies. We start with the SDSS data set. To make the comparison as fair as possible, we rely on the high-luminosity $\left(L \geq L_{*}\right)$, low-space-density redMaGiC sample, as the typical magnitudes of these galaxies are closer to the magnitudes of the galaxies with 

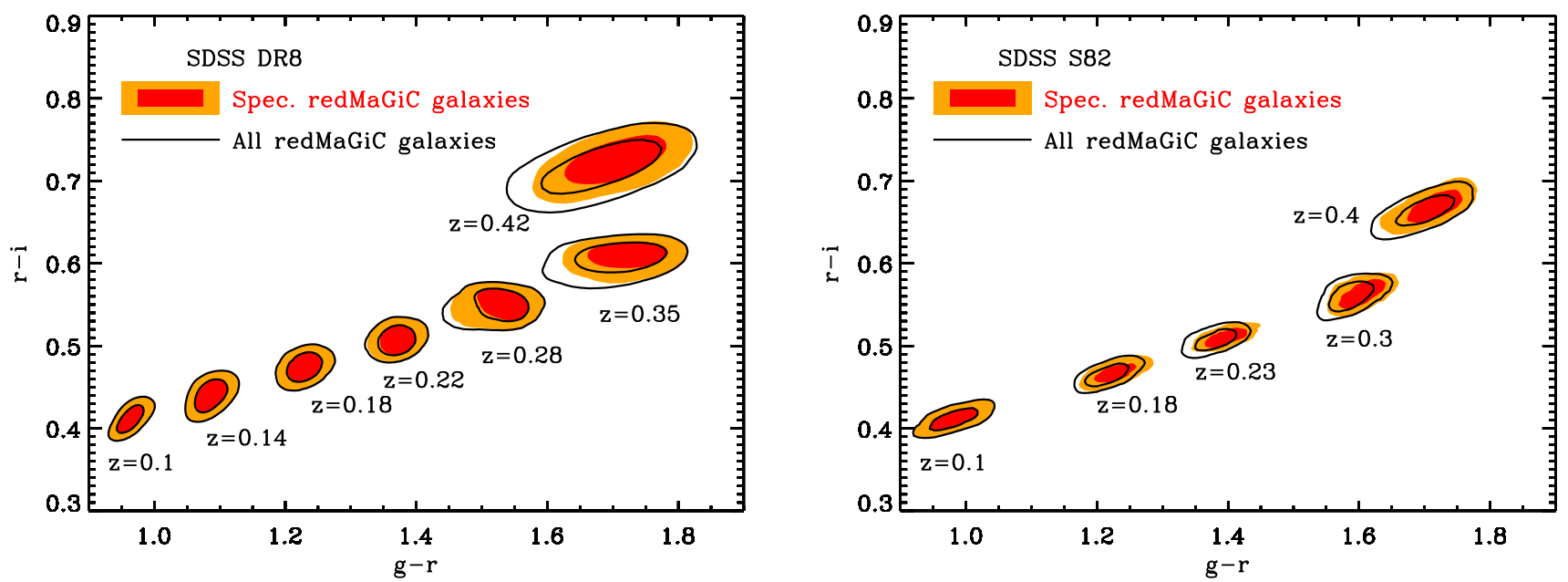

Figure 4. 68 and 95 per cent galaxy density contours in $g-r$ versus $r-i$ space for DR8 and S82 redMaGiC galaxies for a variety of redshift bins, as labelled. Red/orange contours correspond to redMaGiC galaxies with spectroscopic redshifts, while the solid black curves show the contours for the full redMaGiC sample. A mismatch between the coloured and black ellipses implies biased spectroscopic sampling of redMaGiC galaxies.
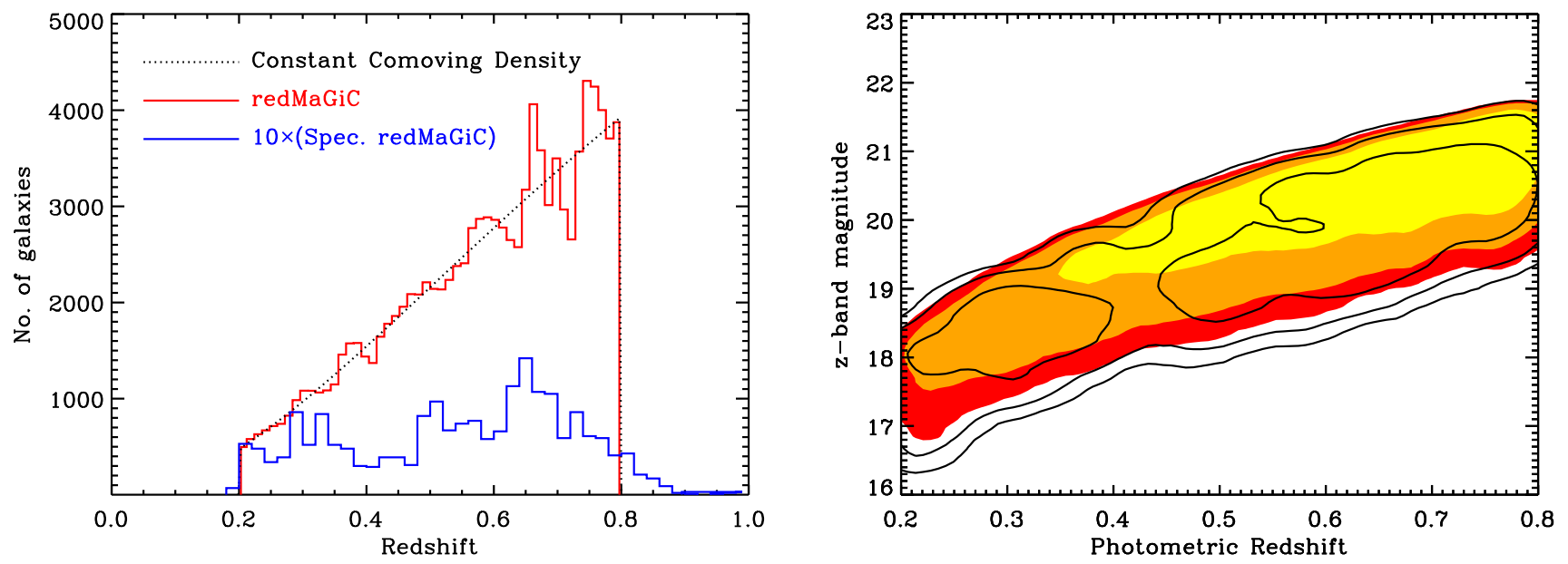

Figure 5. Left: $\mathrm{d} N / \mathrm{d} z$ histogram for the fiducial redMaGiC galaxy sample. The dotted line is the expected distribution for a constant comoving density sample. The red histogram is the redMaGiC data binned by our photometric redshift estimate. The blue histogram shows the number counts for the redMaGiC sample with spectroscopic redshifts, boosted by a factor of 10 for clarity. Right: contours containing 68, 95, and 99 per cent of redMaGiC galaxies (coloured contours) or redMaGiC galaxies with spectroscopic redshifts (solid contours). The dearth of galaxies at $z \approx 0.4$ and the relative excess of bright galaxies in the spectroscopic sample are apparent.

Table 1. Photometric redshift performance of redMaGiC galaxies. All quantities are first computed in redshift bins, and then the median of the binned values is reported. Bias and $|\mathrm{Bias}|$ are the median values for $\left(z_{\text {spec }}-z_{\text {photo }}\right)$ and $\left|z_{\text {spec }}-z_{\text {photo }}\right|$, respectively. The scatter is $1.4826 \times$ MAD, where MAD is the median absolute deviation of $\left|z_{\text {spec }}-z_{\text {photo }}\right| /\left(1+z_{\text {spec }}\right)$. The predicted scatter is the median value of $\sigma_{z} /\left(1+z_{\text {photo }}\right)$, where $\sigma_{z}$ is the reported photo- $z$ error.

\begin{tabular}{llcrcccc}
\hline Space density & Redshift range & Data set & $\operatorname{Bias}(\%)$ & $\mid$ Bias $\mid(\%)$ & Scatter $(\%)$ & Predicted scatter $(\%)$ & $5 \sigma$ outlier fraction $(\%)$ \\
\hline $10^{-3} h^{3} \mathrm{Mpc}^{-3}$ & $z \in[0.2,0.8]$ & DES SV & 0.51 & 0.51 & 1.69 & 1.78 & 1.4 \\
& $z \in[0.1,0.65]$ & SDSS S82 & 0.17 & 0.39 & 1.10 & 0.97 & 1.40 \\
& $z \in[0.1,0.45]$ & SDSS DR8 & -0.04 & 0.20 & 1.43 & 1.50 & 0.8 \\
$2 \times 10^{-4} h^{3} \mathrm{Mpc}^{-3}$ & $z \in[0.2,0.8]$ & DES SV & 0.19 & 0.37 & 1.59 & 1.03 & 1.9 \\
& $z \in[0.1,0.65]$ & SDSS S82 & 0.14 & 0.22 & 1.04 & 1.46 & 1.9 \\
& $z \in[0.1,0.45]$ & SDSS DR8 & -0.22 & 0.22 & 1.40 & & \\
\hline
\end{tabular}

spectroscopic redshifts. Note that this high-luminosity redMaGiC sample goes up to a maximum redshift $z=0.55$ rather than the $z=0.45$ redshift we could achieve with the low-luminosity sample. However, we restrict our attention to $z \in[0.1,0.5]$ rather than $z \in$ $[0.1,0.55]$. This is because for $z \geq 0.5$, the spectroscopic sampling of redMaGiC galaxies becomes increasingly biased, as illustrated in Fig. 6.

We consider three photo- $z$ algorithms. The first set of photo- $z \mathrm{~s}$ are those included with SDSS DR7 (Abazajian et al. 2009), which we shall refer to simply as the SDSS photo-zs. These were obtained 

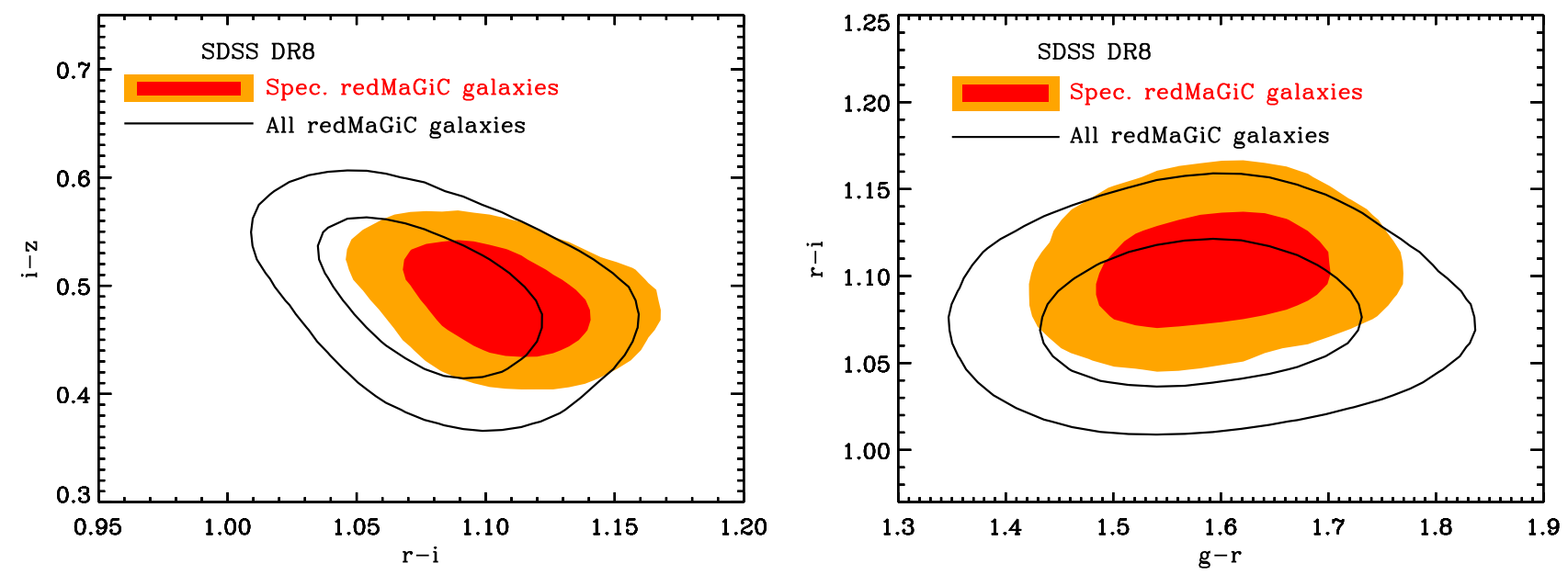

Figure 6. Distribution of redMaGiC galaxies in the photometric redshift bin $z_{\text {photo }} \in[0.54,0.55]$. Orange/red contours show the colour distribution of redMaGiC galaxies with spectroscopic redshifts, while the solid ellipses show the distribution of all redMaGiC galaxies. The large offsets between the two sets of ellipses are due to biased spectroscopic sampling of the redMaGiC galaxies.
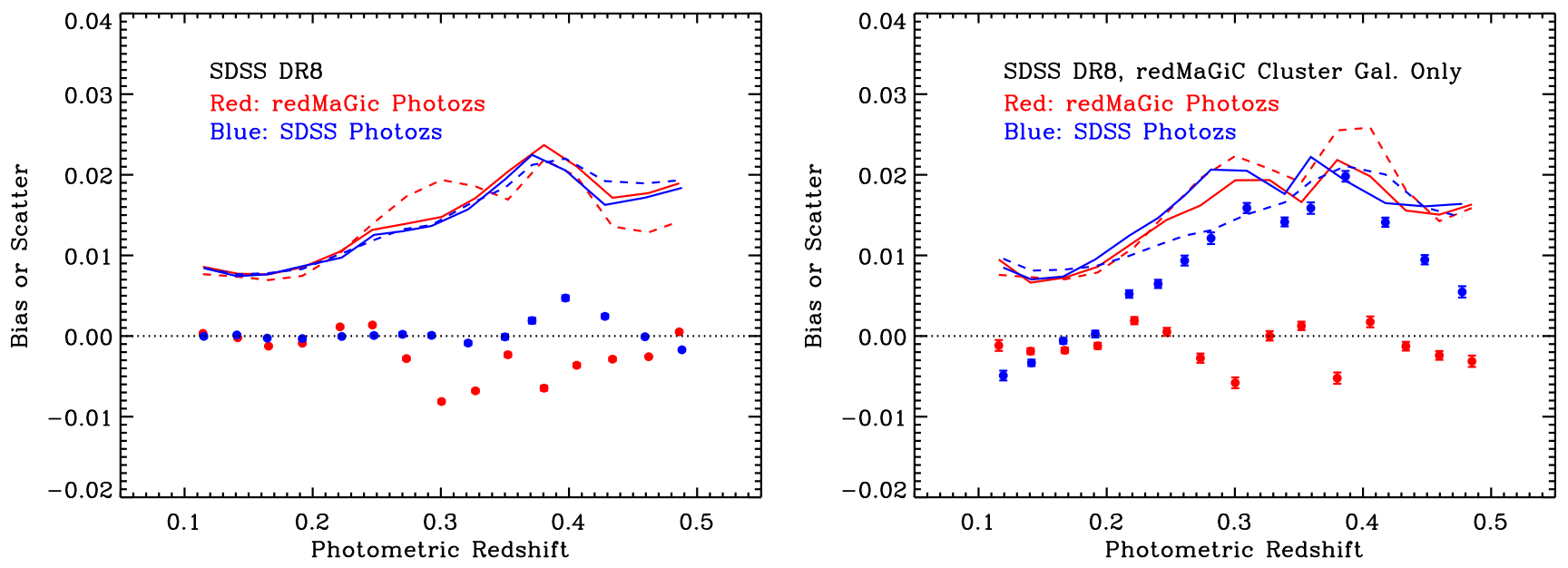

Figure 7. Left: comparison of the photometric redshift performance of redMaGiC (red) and SDSS photo-zs for redMaGiC galaxies (blue). This plot uses SDSS spectroscopic redshifts to compute the redshift bias and scatter of the redMaGiC photo-zs, and is therefore limited to the brightest redMaGiC galaxies. Points with error bars show the median redshift bias for each of the two samples. Solid lines show the observed photo- $z$ scatter, while dashed lines show the predicted scatter. Right: as for the left-hand panel, only now we test the photo- $z$ performance of the subsample of redMaGiC galaxies that are members of redMaPPer clusters. For these galaxies, we assign the photometric redshift of the host redMaPPer clusters as the 'spectroscopic' redshift of the redMaGiC galaxy for the purposes of computing photometric redshift biases and scatter. By doing so, we can test the accuracy of the photometric redshifts of faint redMaGiC galaxies with no spectroscopic redshift.

through a hybrid method that combines the spectral templates of Budavári et al. (2000) with the machine learning method of Csabai et al. (2007). A second set of photo- $z$ s we compare against are those from Hoyle et al. (2015a), which we will refer to as the RDF photo-zs. This algorithm uses a combination of decision trees and feature importance to derive photometric redshift estimates. RDF photo-zs use 85 galaxy features with a $60 / 40$ per cent split for training and validation. Finally, we utilize the publicly available code ANNZ (Collister \& Lahav 2004) to estimate the redshifts of redMaGiC galaxies. This choice is motivated by the results of Abdalla et al. (2011), who performed a detailed comparison of six photometric redshift algorithms, and found ANNZ performed best in LRG samples. We train ANNZ with $2 / 3$ of the full spectroscopic training sample, and test on the remaining $1 / 3$. The neural net had five input nodes (four MODEL_MAG galaxy colours and a total $m_{i}$, for which we use CMODEL_MAG). We utilized two hidden layers of 10 nodes each, as per the standard architecture.

A comparison of the redMaGiC photo- $z$ to the SDSS photo- $z \mathrm{~s}$ is shown in Fig. 7. We find that the SDSS photo-zs are slightly less biased than the redMaGiC photo- $z \mathrm{~s}$, but have nearly identical scatters. The SDSS photo- $z$ s also do a better job of error characterization, though the difference is not large. The picture is much the same for ANNZ, except that ANNZ grossly underestimates the photometric redshift scatter (not shown). RDF redshifts are clearly superior to the SDSS, ANNZ, and redMaGiC photo- $z$ s, though the improvement remains modest: the scatter decreases from 1.48 per cent in redMaGiC to 1.28 per cent in RDF (not shown). The agreement between the ANNZ, SDSS, and redMaGiC redshifts strongly suggests that the improvement seen with RDF is primarily due to the large number of features used ( 85 observables), rather than more 
Table 2. As Table 1, but comparing the redshift performance of different photo- $z$ algorithms on redMaGiC galaxies. We only consider the redMaGiC sample with space density $2 \times 10^{-4} h^{3} \mathrm{Mpc}^{-3}$. The redshift range of consideration is $z \in[0.1,0.5]$ for DR8, and $[0.2,0.8]$ for DES. 'Bad fraction' is the fraction of galaxies where $\left|z_{\text {photo }}-z_{\text {spec }}\right| /\left(1+z_{\text {spec }}\right) \geq 0.07$ (for SDSS) or $\geq 0.08$ (for DES), corresponding roughly to $5 \sigma$ for redMaGiC photo-zs. DR8 Spec AB data sets correspond to redMaGiC with a spectroscopic afterburner (see Section 7).

\begin{tabular}{lrcccr}
\hline Data set & Bias $(\%)$ & $\mid$ Bias $\mid(\%)$ & Scatter(\%) & Predicted scatter(\%) & Bad fraction(\%) \\
\hline SV redMaGiC & 0.35 & 0.35 & 1.82 & 1.80 & 1.4 \\
SV SKYNET & -0.36 & 0.59 & 1.58 & 5.31 & 1.1 \\
SV BPZ & 1.48 & 2.95 & 1.59 & 9.821 & 11.6 \\
DR8 redMaGiC & -0.23 & 0.23 & 1.48 & 1.39 & 1.4 \\
DR8 SDSS photo- $z$ & -0.00 & 0.02 & 1.37 & 1.38 & 1.3 \\
DR8 RDF photo- $z$ & 0.01 & 0.03 & 1.25 & 1.28 & 1.3 \\
DR8 ANNZ photo- $z$ & -0.09 & 0.13 & 1.33 & 1.29 & 1.5 \\
DR8 Spec AB & 0.01 & 0.03 & 1.49 & 1.47 & 1.1 \\
\hline
\end{tabular}

optimal use of the limited information used in redMaGiC (five bands).

A quantitative summary of these results is presented in Table 2 (along with summary of the results from the next section). Also reported there are the fraction of galaxies where $\left|z_{\text {photo }}-z_{\text {spec }}\right| /(1+$ $\left.z_{\text {spec }}\right) \geq 0.07$, corresponding roughly to $5 \sigma$ for redMaGiC galaxies. This number characterizes how large the tails of the photo- $z$ errors are. All methods we consider here have comparable tails.

We caution, however, that these tests represent a best-case scenario for training set methods. Specifically, machine learning methods do not extrapolate outside their training sets very well. Consider red galaxies as a specific example. Because the red sequence is tilted, a faint red-sequence galaxy will appear bluer than a bright red-sequence galaxy. Consequently, red-sequence galaxies fainter than the training data set of a machine learning algorithm will have $z_{\text {photo }} \leq z_{\text {spec }}$.

We can indirectly verify this expectation by looking at members of galaxy clusters. Specifically, we select all redMaPPer highprobability (membership probability $\geq 90$ percent) cluster members, and assign to all such members a 'spectroscopic' redshift equal to the photometric cluster redshift. We then compare the redMaGiC and SDSS photo- $z$ s of these galaxies to their assigned cluster redshifts. The redshift bias $z_{\text {cluster }}-z_{\text {photo }}$ and corresponding scatter are shown in the right-hand panel of Fig. 7. We see that our expectation that $z_{\text {photo }} \leq z_{\text {spec }}$ is borne out by the data, and that the bias can be large, $\approx 0.02$. At very high redshifts, the luminosity threshold in redMaPPer approaches the spectroscopic magnitude limit, and so the bias starts to decrease with redshift.

The main takeaways from these tests are that redMaGiC photo$z \mathrm{~s}$ perform as well as the best machine learning methods run with the same photometric input. However, machine learning methods can improve on redMaGiC by exploiting additional data. Critically, however, machine methods do not extrapolate well, and appear to be subject to large redshift biases for galaxies that are not well represented in the training data sets (however, see Hoyle et al. 2015b). Because of how the redMaGiC algorithm is structured, this is not a problem for redMaGiC photo- $z$ s.

\subsection{DES comparisons}

We compare redMaGiC photo-zsto two algorithms currently in use within the DES collaboration (Sánchez et al. 2014), specifically SKYNET and BPZ photo- $z$ S. SKYNET is a machine learning method that relies on neural networks to 'classify' galaxies into redshift bins (Graff et al. 2014; Bonnett 2015), while BPZ is a popular templatebased code (Benítez 2000). We use BPZ with its default configuration

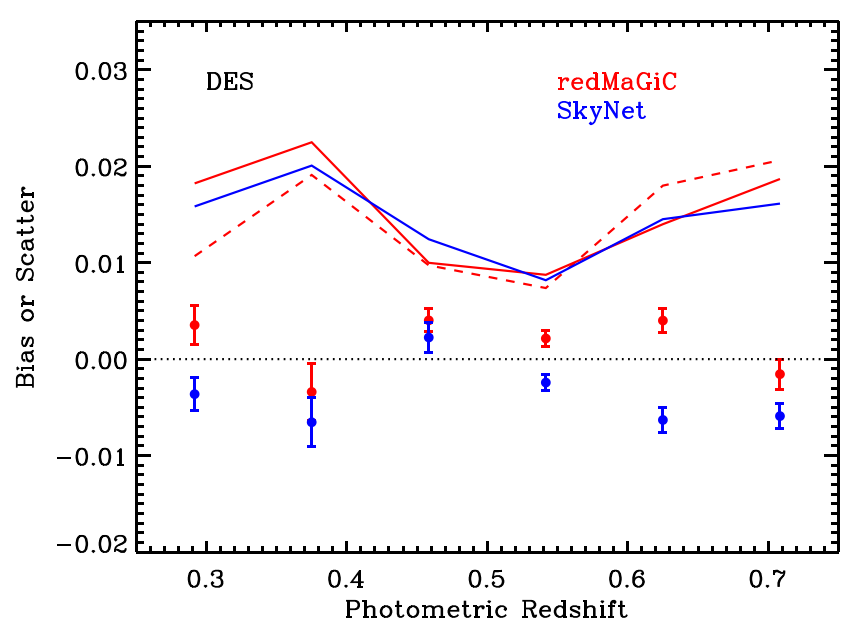

Figure 8. As Fig. 7, only now we compare SV SKYNET photo-zs (blue) to SV redMaGiC photo-zs(red). The predicted SKYNET scatter is not shown, as the SKYNET predicted errors are a factor of 3.5 larger than the observed scatter. That is, the putative dashed blue line showing the predicted SKYNET scatter is off the scale in the above plot, demonstrating poor agreement between the actual and predicted performance of the SKYNET photo- $z$ s on the redMaGiC galaxies.

(eight templates, INTERP $=\mathbf{2}$, and we do not allow for zero-point offsets). While there are other machine learning methods available in DES, they all have comparable performance, so we have arbitrarily chosen to focus on SKYNET to simplify our analysis.

Fig. 8 compares the performance of SKYNET on the redMaGiC galaxy sample to that of the redMaGiC photo-zs. The two algorithms perform equally well in terms of photo- $z$ biases and scatter. However, SKYNET grossly overestimates the photometric redshift uncertainty, with the SKYNET predicted uncertainties being a factor of 3.5 larger than the observed errors. This is not unexpected: SKYNET and the other machine learning codes used in the DES SV data have their photometric redshifts smoothed and broadened (for details, see appendix $C$ in Bonnett et al. 2015), which improves photo- $z$ performance for lensing sources, but, as evidenced here, has a deleterious effect on the photo- $z$ error estimates for redMaGiC galaxies. SKYNET and redMaGiC also exhibit similar tails.

BPZ performs very poorly at low redshifts, exhibiting a redshift bias of $\approx 0.1$. The bias decreases to $\approx 0.02$ at higher redshifts, but remains well above the SKYNET/redMaGiC biases. The redshift scatter for BPZ is comparable to that of SKYNET/redMaGiC, but the uncertainties are overestimated by a factor of $\approx 6$. Nearly 12 per cent of all 
galaxies have $\left|z_{\text {spec }}-z_{\text {photo }}\right| /\left(1+z_{\text {spec }}\right) \geq 0.08$ for BPZ, compared with $\approx 1.4$ per cent for redMaGiC/SKYNET.

Our results confirm the basic picture we obtained from the DR8 comparisons: redMaGiC performs as well as the best performing machine learning methods, despite not requiring representative spectroscopic training samples. BPZ performance is especially poor. Importantly, redMaGiC continues to have extremely well characterized scatter, whereas SKYNET/BPZ do not.

\section{WHY SELECTION MATTERS}

The primary motivation of the redMaGiC algorithm is not to improve upon existing photometric redshift algorithms, but rather to select a galaxy sample with robust photo-zs. The results in the previous section clearly demonstrate that redMaGiC galaxies do, in fact, have photometric redshifts that are both precise and accurate. In this section, we investigate whether this feature is unique to the redMaGiC sample. In particular, we look at the current workhorse for LSS measurements in the SDSS, the CMASS galaxy sample. CMASS galaxies were specifically selected to be roughly stellar mass limited at $z \geq 0.45$. Here, we test whether the redMaGiC selection can lead to improved photometric redshift performance relative to CMASS. Note that any gains we make are not of critical importance for spectroscopic experiments, as such experiments are not sensitive to large photometric redshift scatter and/or catastrophic photo- $z$ failures.

A fair comparison of CMASS to redMaGiC galaxies is difficult. In particular, we would like to compare samples that have comparable space densities (which control the errors in clustering signal) and luminosities (which set the photometric error uncertainty). For comparison purposes, Table 3 quotes typical densities for a couple of standard SDSS galaxy samples, namely LRG (Eisenstein et al. 2001), and LOWZ and CMASS (Dawson et al. 2013) Also shown is the minimum luminosity of galaxies in that sample at a typical redshift. Densities for the standard SDSS samples are based on fig. 1 of Tojeiro et al. (2014). We see that even our bright redMaGiC sample has a comparable density to CMASS, but a lower luminosity threshold, reflecting the more stringent colour cuts applied in redMaGiC. We will compare CMASS against this sample. Note that CMASS galaxies are $\approx 0.3$ mag brighter than the redMaGiC galaxies we

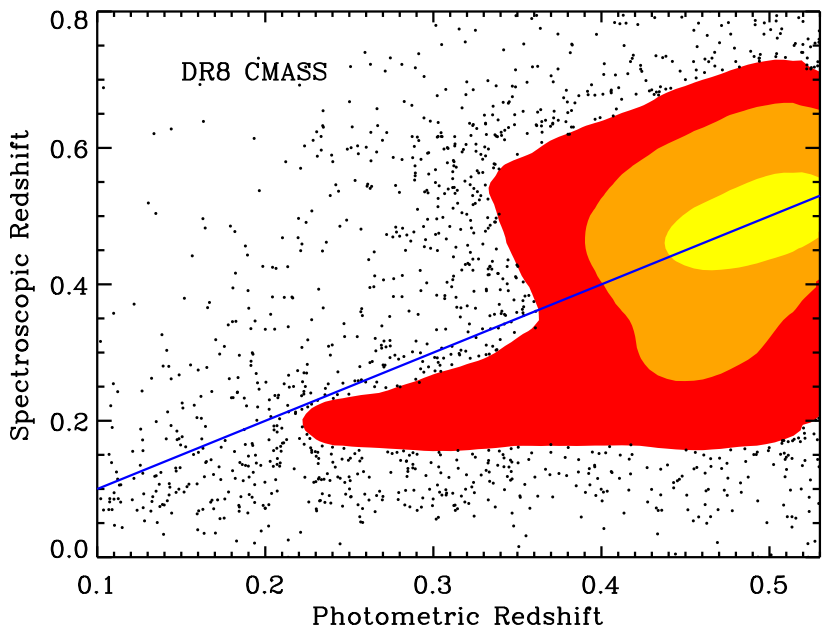

Table 3. Typical space density and luminosity cuts for a variety of different SDSS galaxy samples.

\begin{tabular}{lcc}
\hline Sample & $\begin{array}{c}\text { Space density } \\
\left(h^{-1} \mathrm{Mpc}^{-3}\right)\end{array}$ & $\begin{array}{c}\text { Minimum luminosity } \\
\left(L_{\min } / L_{*}\right)\end{array}$ \\
\hline LRG & $1 \times 10^{-4}$ & $2.1($ at $z=0.35)$ \\
LOWZ & $3 \times 10^{-4}$ & $1.6($ at $z=0.35)$ \\
CMASS & $2 \times 10^{-4}$ & $1.5($ at $z=0.5)$ \\
redMaGiC Bright & $2 \times 10^{-4}$ & 1.0 \\
redMaGiC Faint & $1 \times 10^{-3}$ & 0.5 \\
\hline
\end{tabular}

compare against. This added noise should degrade the photometric redshift performance in redMaGiC galaxies relative to CMASS. That is, the match-up is purposely stacked against redMaGiC for this comparison.

Fig. 9 shows how galaxies fall in the $z_{\text {spec }}-z_{\text {photo }}$ plane for both CMASS (left-hand panel) and redMaGiC (right-hand panel). For the CMASS data set, we rely on SDSS photo-zs (Csabai et al. 2007), while we use redMaGiC photo- $z$ s for redMaGiC. Note that redMaGiC and SDSS photo- $z$ s had nearly identical performance on redMaGiC galaxies, so the performance in the right-hand plot would be much the same if we replaced redMaGiC photo- $z$ s with SDSS photo-zs.

The benefit of the redMaGiC selection is immediately apparent: despite probing fainter galaxies, the redMaGiC galaxies have clearly better behaved photometric redshifts than those of CMASS. The photo- $z$ scatter is 1.5 per cent for redMaGiCand 2.1 per cent for CMASS. In addition, the fraction of galaxies with large redshift errors $(|\Delta z| /(1+z) \geq 0.07)$ is much larger for CMASS (6.4 per cent) than for redMaGiC (1.4 per cent). We note that the photo- $z$ scatter for CMASS galaxies quoted here is significantly lower than that reported in Ross et al. (2011). This is partly because we define scatter as $\sigma_{z} /(1+z)$, while Ross et al. (2011) quote $\sigma_{z}$, and partly because we estimate $\sigma_{z}$ using median statistics, while Ross et al. (2011) use $\sigma_{z}=\sqrt{\left\langle z_{\text {spec }}-z_{\text {photo }}\right\rangle^{2}}$, which is more sensitive to gross outliers than the MAD-based estimate.

It is also clear from Fig. 9 that CMASS galaxies with $z_{\text {spec }} \lesssim 0.3$ are particularly ill-behaved. This is not really problematic for experiments like BOSS, where the spectroscopic follow-up of the targets ensures that these contaminants do not percolate into

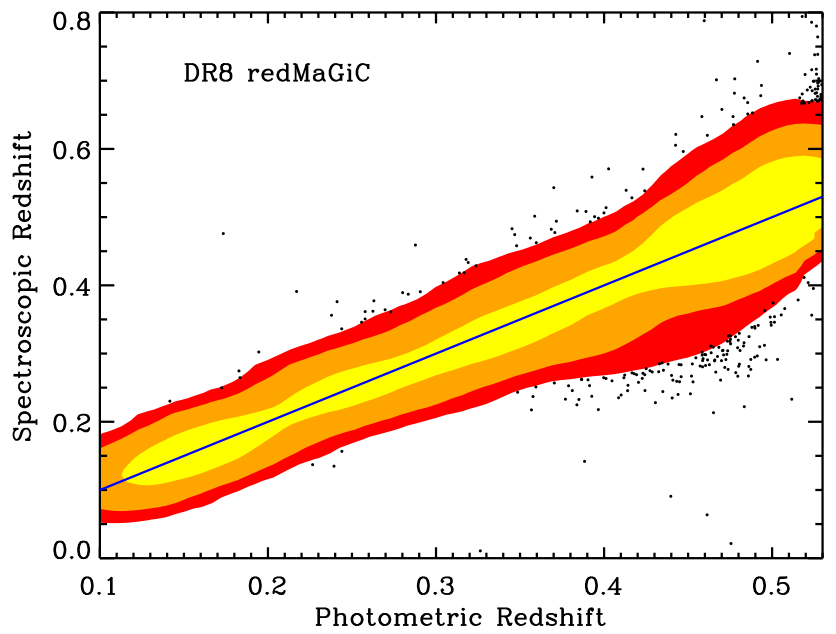

Figure 9. Left: spectroscopic versus photometric redshifts for CMASS galaxies using SDSS photo- $z$ s. Coloured regions contain 68 , 95 , and 99 per cent of the points. The remaining 1 per cent of galaxies are shown as points. The blue line is the $y=x$ line. Right: as the left-hand panel, but for redMaGiC galaxies. 

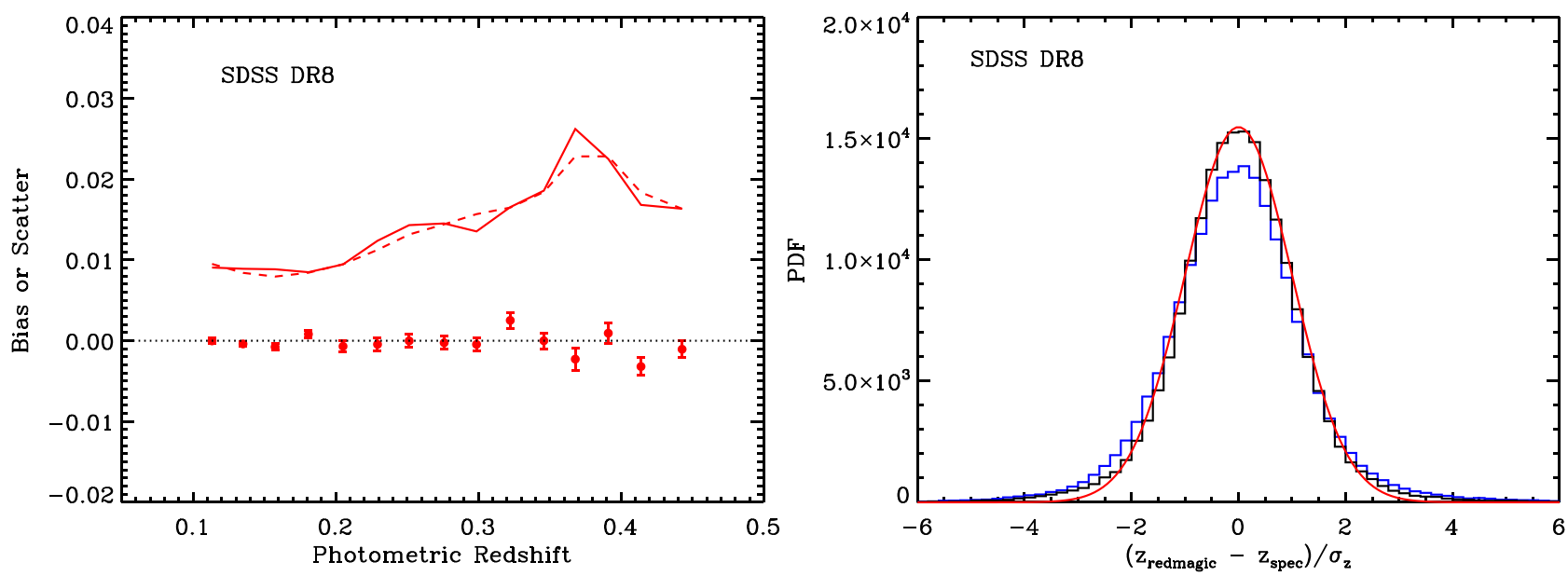

Figure 10. Left: redMaGiC photometric redshift performance after training with a spectroscopic subsample of galaxies. Points with error bars show the bias in the recovered redshifts. The solid line shows the photometric redshift scatter, while the dashed line shows the predicted redshift scatter. Right: a histogram of the quantity $\Delta=\left(z_{\mathrm{spec}}-z_{\text {photo }}\right) / \sigma_{z}$, where $\sigma_{z}$ is the reported photometric redshift uncertainty. The blue histogram is for our fiducial redMaGiC sample, while the black histogram is for a spectroscopically trained redMaGiC sample. The red curve is not a fit. It is simply a Gaussian of zero mean and unit variance.

cluster measurements at $z \approx 0.5$. By contrast, a photometric survey would end up including those galaxies in its clustering measurements, leading to systematic errors in the clustering signal. This further highlights the importance of redMaGiC selection for photometric LSS studies.

We can also compare the performance of the RDF photometric redshifts in the CMASS sample to redMaGiC. Relative to the SDSS photo-zs, RDF shows clear improvement: the scatter is reduced to 1.9 per cent, and the fraction of galaxies with larger errors goes down to 2.2 per cent. This is not surprising: RDF redshifts were trained on CMASS galaxies, whereas the SDSS photo- $z$ s were not. This highlights the importance of training for machine learning methods, a weakness not shared by redMaGiC. Just as importantly, even RDF redshifts for CMASS galaxies are worse than redMaGiC redshifts for redMaGiC.

In short, we find that redMaGiC is extremely successful at identifying galaxies with robust photometric redshift estimates. Of course, CMASS was designed to be used for a spectroscopic survey, so the differences highlighted here are much less important in that case. For purely photometric surveys, however, our selection algorithm is clearly superior.

\section{SPECTROSCOPIC TRAINING OF redMaGiC}

We consider whether $z_{\mathrm{rm}}$ from redMaGiC can be significantly improved with further spectroscopic training data. Specifically, in the redMaGiC algorithm, we use a photo- $z$ 'afterburner' that relies on photometric cluster galaxies to help fine-tune our photo- $z$ s. We now consider what happens if we apply a further 'afterburner' using spectroscopic redshift information for the redMaGiC sample. As a proof of concept, we use the redMaGiC galaxies that are in the SDSS DR10 spectroscopic catalogue, and split the sample into half for training and validation. All results shown are for the validation sample only.

For our spectroscopic afterburner, we apply the same procedure as outlined in Section 3.4, only now the initial redshift estimate is $z_{\mathrm{rm}}$. We label our final redshift estimate $z_{\mathrm{sAB}}$ (for spectroscopic afterburner). Similarly, we tweak the photo- $z$ error using median statistics as with our original afterburner. Having defined our new redMaGiC spectroscopically trained photo- $z$ estimates, we test the
redMaGiC photo- $z$ performance using our test sample. The results are shown in the left-hand panel of Fig. 10. The right-hand panel of Fig. 10 shows a histogram of the quantity $\Delta_{z}=\left(z_{\mathrm{spec}}-z_{\mathrm{sAB}}\right) / \sigma_{z_{\mathrm{sAB}}}$. If all the photo- $z$ s were Gaussian, unbiased, and we correctly estimated the photo- $z$ error, then a histogram of the quantity $\Delta_{z}$ would be well fitted by a Gaussian of zero mean and unit variance. The right-hand panel of Fig. 10 shows the $\Delta_{z}$ histogram for the redMaGiC testing sample. The red Gaussian is not a best fit: it is a Gaussian of zero mean and unit variance.

Given the improved performance for the spectroscopically trained redMaGiC sample, why do we not adopt this procedure as part of the redMaGiC photometric redshift estimate by default? As discussed in Section 4, biased spectroscopic sampling of our data set will introduce unknown and uncontrolled biases in the resulting photometric redshifts. Consequently, we have opted not to apply this spectroscopic afterburner until a fully representative spectroscopic galaxy sample becomes available, or data augmentation techniques are advanced enough to extrapolate outside the training sets.

\section{UNDERSTANDING redMaGiC OUTLIERS}

We now investigate the photo- $z$ outliers in the redMaGiC galaxy sample. We consider a galaxy an outlier if its photo- $z$ is more than $5 \sigma$ away from its spectroscopic redshift. The outlier fraction of redMaGiC galaxies as a function of redshift is illustrated in Fig. 11 for both the fiducial and high-luminosity samples. Perhaps the two most salient features in this plot are (1) the difference in the outlier fractions at low redshifts between the SDSS DR8 and both the SDSS S82 and DES SV data sets; and (2) the difference in the outlier fractions between the fiducial and high-luminosity galaxy samples. The latter result is not surprising: the brighter the galaxy, the easier it is to distinguish between red-sequence and non-redsequence galaxies. We will return to the difference between the DR8 and S82/DES SV momentarily.

Consider first the DR8 outlier population. The mean DR8 outlier fraction is small, $\approx 0.7$ percent, and is split among three sets of outlier clumps, as seen in Fig. 3. This last one is more readily apparent in the SDSS S82 data set. We consider each of these in turn. 

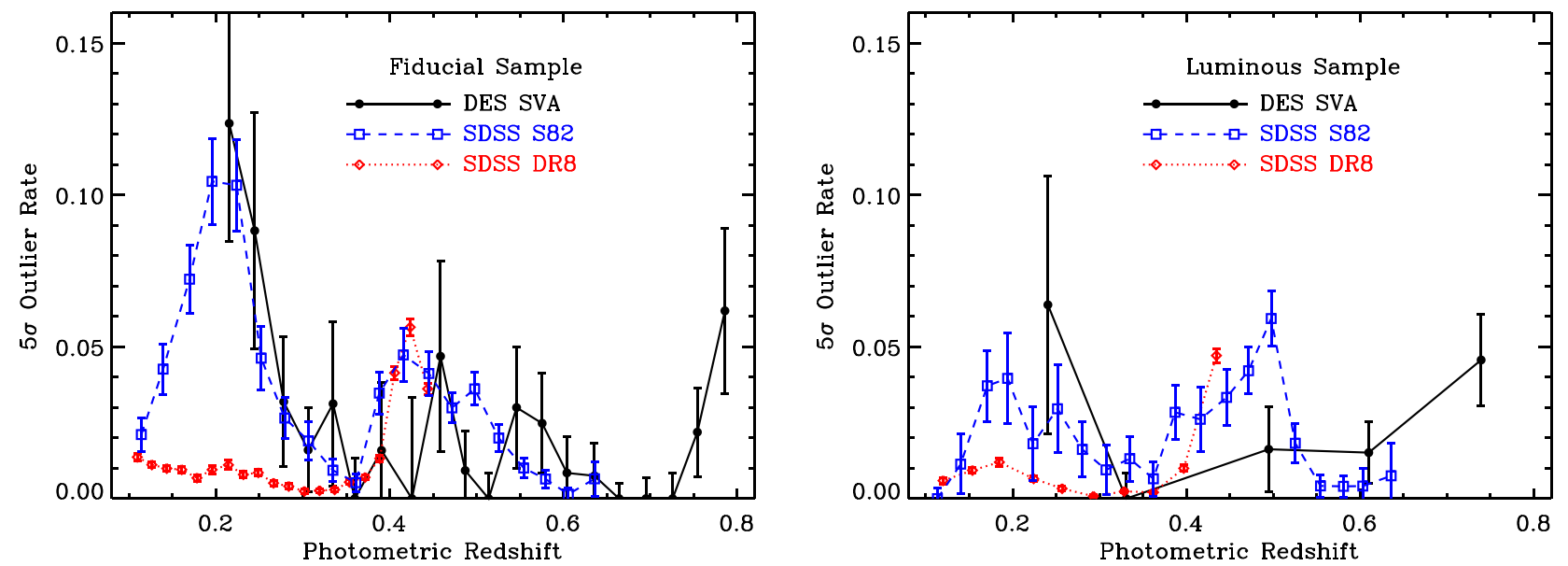

Figure 11. $5 \sigma$ outlier fraction for the fiducial and high-luminosity DR8 (red), S82 (blue), and DES (black) redMaGiC samples as estimated using SDSS DR12 spectroscopy.

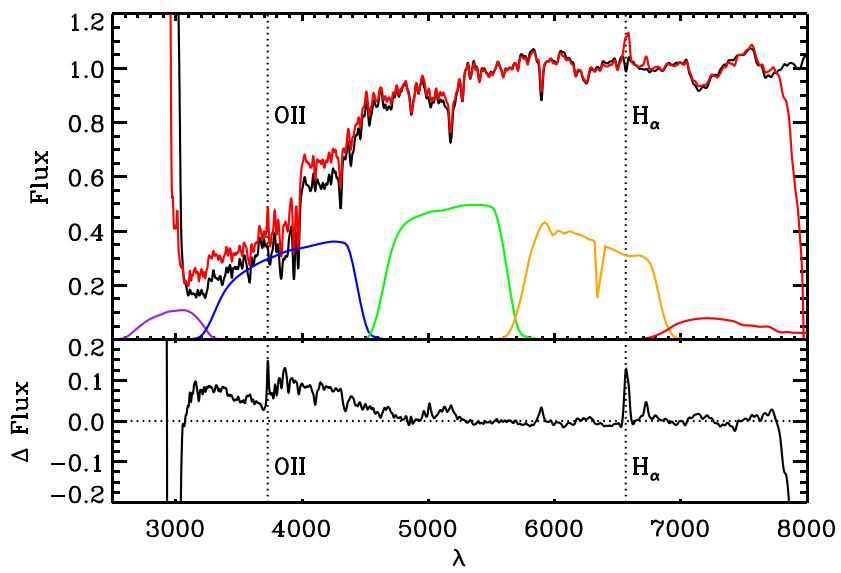

Figure 12. Top panel: stacked rest-frame spectra for redMaGiC galaxies with $z_{\text {photo }} \in[0.18,0.22]$. Outlier galaxies are shown in red (Clump 1 in Fig. 3), and non-outliers in black. Also shown are the SDSS ugriz transmission curves for an extended source at $z=0.2$ assuming 1.3 airmasses (purple, blue, green, orange, red). Bottom panel: difference between the two spectra in the top panel, showing the excess emission associated with the outlier galaxy population. The vertical dotted lines mark the [O II] (leftmost line) and $\mathrm{H} \alpha$ (rightmost line) emission lines. Clump 1 galaxies have excess blue light, as well as [O II] and $\mathrm{H} \alpha$ emission indicative of a small amount of residual star formation.

\subsection{Clump 1: low-redshift outliers}

We compare the rest-frame spectra of outliers in Clump 1 (the low-redshift outliers in Fig. 3) to a control sample of non-outliers. The control sample is comprised of galaxies with good photo- $z \mathrm{~s}$ (within $0.5 \sigma$ of $z_{\text {spec }}=z_{\text {photo }}$ ). We randomly sample from the control sample so as to mirror the photo- $z$ distribution of the outlier sample. We median-stack the spectra of both sets of galaxies, arbitrarily normalizing them to unity over the wavelength range $\lambda=$ [5300 ̊, $5800 \AA$ ]. We have further smoothed the spectra to make the resulting stacks easier to interpret by eye. The two stacked spectra and their difference are shown in Fig. 12.

We find that the two spectra are largely consistent with each other for wavelengths $\lambda \gtrsim 5000 \AA$. At shorter wavelengths, however, there is a clear excess of blue light in the photometric redshift outliers. In addition, the spectra of the outlier galaxies have obvious $\mathrm{H} \alpha$ and

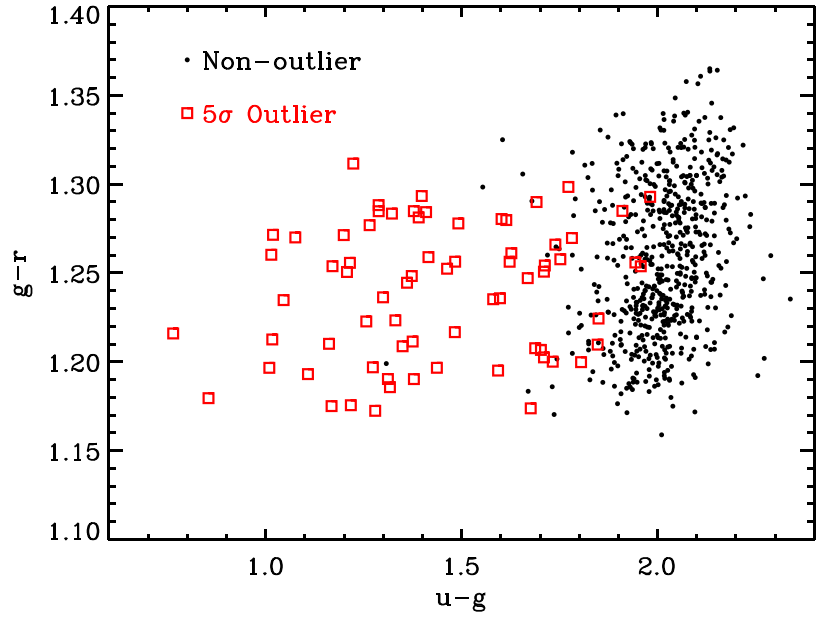

Figure 13. Distribution of our fiducial $\mathrm{S} 82$ redMaGiC galaxy sample in $u-g$ and $g-r$ space for galaxies in the photometric redshift bin $z_{\text {photo }} \in[0.18,0.22]$. Black points are galaxies where our photometric redshift estimate agrees with the spectroscopic estimate within $2 \sigma$, while red points correspond to $\geq 5 \sigma$ redshift outliers.

[O II] lines, demonstrating that these galaxies have ongoing star formation.

Why is the fraction of outliers in Clump 1 so much larger in S82 and SV data sets relative to the DR8 sample? This is because the S82 and SV redMaGiC selection was based solely on griz photometry, while for DR8 we additionally included $u$-band photometry. As the $u$ band is sensitive to the enhanced star formation in Clump 1 galaxies, the relative contamination of these outliers is dramatically decreased in DR8 relative to the S82 and SV data sets. While we did not use the S82 $u$ band in the construction of the redMaPPer and redMaGiC catalogues - its inclusion created problems with the higher redshift $(z \sim 0.5)$ cluster calibration - we do have the data available for us to test our hypothesis. Fig. 13 shows $\mathrm{S} 82$ redMaGiC galaxies in the photometric redshift slice $z_{\text {photo }} \in[0.18,0.22]$. Black points are galaxies where the spectroscopic redshift of the galaxy is within $2 \sigma$ of our photometric estimate, while red points show $\geq 5 \sigma$ outliers. We see that the vast majority of $5 \sigma$ outliers are unusually bright in $u$, as expected. 


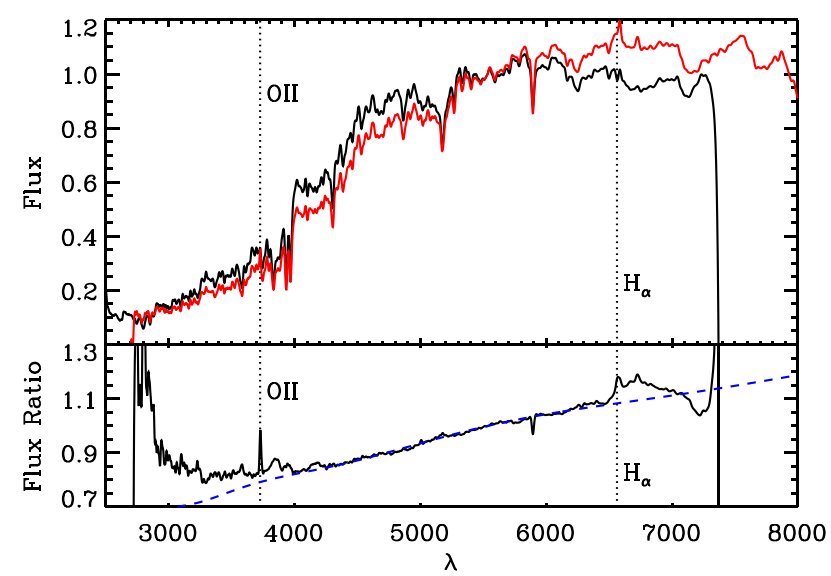

Figure 14. Top panel: stacked rest-frame spectrum of outlier (red) and nonoutlier (black) redMaGiC galaxies for Clump 2 (see Fig. 3). Bottom panel: ratio of the outlier to non-outlier spectra (black line). The dashed blue line shows the effects of internal dust reddening with $E(B-V)=0.15$. The vertical dotted lines mark the [O II] and $\mathrm{H} \alpha$ emission lines, indicating a small amount of residual star formation, as with the Clump 1 galaxies.

\subsection{Clump 2: photo- $z$ biased high}

We repeat the spectral-stacking procedure above for Clump 2 galaxies (with photo- $z$ biased high in Fig. 3). For reasons that will become apparent below, in Fig. 14 we plot not the difference between the outlier and non-outlier spectra, but rather their ratios. Both sets of spectra have been normalized as before. A blue light excess is immediately apparent, and we again see both $\mathrm{H} \alpha$ and [O II] emission. However, the most salient feature is the slope of the flux ratio as a function of wavelength, with the outlier spectra having a systematically steeper continuum than the non-outlier galaxies. This slope is consistent with internal dust reddening in the galaxy. Specifically, the dashed blue line is the predicted spectral ratio assuming an O'Donnell (1994) reddening law with $E(B-V)=0.15$.

The reasons why these dusty galaxies show up in our redMaGiC selection only at this particular redshift range are worth noting. In particular, at most redshifts the rest-frame reddening vector with broad-band griz photometry is not parallel to the colour evolution vector of the red sequence. Consequently, at most redshifts a galaxy that starts in the red sequence and is reddened simply moves off the red sequence, and is not selected. By contrast, at $z \approx 0.35$, the rest-frame reddening vector is parallel to the colour evolution vector of red sequence, so dust reddening can move a galaxy from $z_{\text {spec }} \sim 0.3$ to $z_{\text {photo }} \sim 0.4$. At the same time, the internal reddening will suppress the excess blue emission, reducing excess blue light as a discriminator for these galaxies. It should also be noted that internal reddening also dims the galaxy, and thus tends to increase photometric errors, making it even more difficult to distinguish these galaxies from the expected template.

\subsection{Clump 3: photo- $z$ biased low}

Finally, we repeat our spectral-stacking procedure for Clump 3 galaxies (with photo- $z$ biased low in Fig. 3). In Fig. 15, we show the difference between the outlier and non-outlier spectra (black line). As a comparison, we show the difference between outliers and nonoutliers for Clump 1 (red dashed line), which are similar in that they have $z_{\mathrm{rm}}$ biased low. We see that the differences are qualitatively similar, but that the Clump 3 galaxies have excess emission that

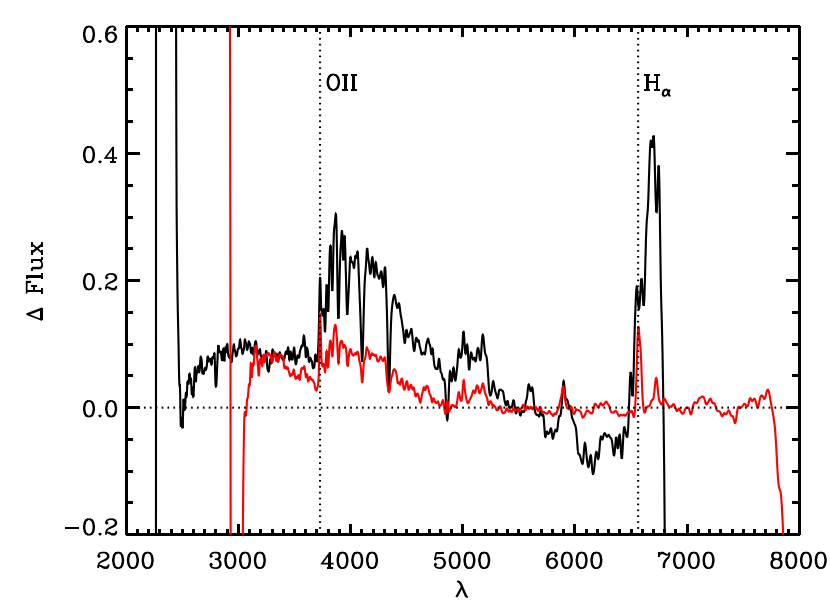

Figure 15. Difference between the outlier and non-outlier stacked restframe spectra for Clump 1 (red) and Clump 3 (black) galaxies (see Fig. 3). The vertical dotted lines mark the [O II] (leftmost line) and $\mathrm{H} \alpha$ (rightmost line) emission lines. Clump 3 galaxies are qualitatively similar to those in Clump 1, with residual star formation that is not large enough to drive the galaxy from the photometric red sequence at SDSS depths.

is significantly larger than that of Clump 1. This makes sense, as the SDSS DR8 imaging is relatively shallow, and therefore the small photometric errors for Clump 1 galaxies make the redMaGiC selection more efficient. In contrast, at higher redshifts, the larger photometric errors allow for a larger excess emission.

Having identified the physical origin of the various outlier populations of redMaGiC galaxies, it may be possible to construct observables that allow us to reject such galaxies from the redMaPPer sample. We leave an exploration of this possibility to future work. Of course, it may be possible that some of the outlier populations cannot be removed with the available photometry. For instance, we expect that Clump 1 outliers in the DES will be difficult to remove without $u$ band. If these outlier populations are irreducible, then they must be adequately characterized and the corresponding $P(z)$ distributions for the redMaGiC galaxies must be correspondingly updated. Alternatively, the corresponding redshift regions ought to be excluded from high-precision LSS studies.

\section{SUMMARY AND CONCLUSIONS}

Photometric redshift systematics are the primary challenge that must be overcome for pursuing LSS studies with photometric data sets. Based on the fact that red-sequence galaxies tend to have excellent photometric redshifts, we have sought to address this challenge by refining red-sequence selection algorithms in the hope of creating a 'gold' photometric galaxy sample for photometric LSS studies. A complementary goal is to develop a new photometric redshift estimator for these galaxies. The result is the redMaGiC algorithm.

Conceptually, the algorithm is exceedingly simple: one specifies a desired comoving space density and luminosity threshold. The algorithm then fits all galaxies with a red-sequence template and assigns the galaxies a redshift. Based on these redshifts, we apply the desired luminosity threshold. Finally, we then keep rank-order galaxies by the goodness-of-fit statistic $\chi^{2}$, and keep the top $N$ galaxies that lead to the desired comoving space density. In practice, the algorithm is necessarily more difficult to implement due to coupling of the photometric redshift estimates to the galaxy density 
via the photometric redshift afterburner, but the above description captures the spirit of the algorithm well.

We note one important consequence of how we have chosen to define the redMaGiC sample, namely our demand that the comoving density of the galaxies be approximately constant. By enforcing this requirement, it follows that the redMaGiC sample cannot be straightforwardly used to study the evolution of galaxy density as a function of redshift. However, one could still constrain models of evolution of the galaxy luminosity function. In either case, however, we caution that redMaGiC galaxies are necessary an incomplete, non-representative sample of all red galaxies. Indeed, our galaxies are specifically selected to have clean photo-zs, and thus have a sharp (photometric error dependent) cut around red-sequence galaxy colours. This selection effect likely severely compromises the utility of our galaxy sample for at least some subset of galaxy evolution studies. This is a compromise we have taken for the purpose of maximizing the utility of this galaxy sample for LSS studies.

As shown in Section 4, we find that redMaGiC is indeed successful at identifying red-sequence galaxies, and that the corresponding photometric redshift estimates are of very high quality, with a low bias $(\lesssim 0.5$ per cent $)$, low scatter $\lesssim 1.6$ per cent, and low rate of catastrophic outliers $\leq 2$ per cent, with the exact values depending on the precise sample under consideration. As demonstrated in Section 6, the redMaGiC selection yields galaxies with superior photo- $z$ performance to the standard colour-cut selection method used to define the SDSS CMASS sample. In addition, the photo$z$ scatter is correctly estimated a priori. As detailed in Section 5, this performance is comparable to the best machine learning photo$z$ algorithms available today when the same input data are used. Machine learning algorithms can improve upon the photo- $z$ performance of redMaGiC if additional information is provided, though the improvement remains modest.

There are, however, two critical advantages of redMaGiC photo$z \mathrm{~s}$ relative to machine learning-based algorithms. The first is that redMaGiC has minimal spectroscopic requirements: it is much easier to get the necessary cluster redshifts that enable the redMaGiC algorithm than it is to acquire representative training samples for redMaGiC. The second important difference is that, in the absence of representative spectroscopic sampling, machine learning-based algorithms are expected to be biased for galaxies that fall outside the training data set, especially at the faint end as demonstrated in Fig. 7. This failure mode is non-existent for redMaGiC.

Of course, should representative spectroscopic training sets become available for redMaGiC galaxies in the future, one should pursue machine learning techniques to improve redMaGiC photozs. Even with the context of redMaGiC, we explicitly demonstrated that representative spectroscopic sampling of redMaGiC galaxies enables photo- $z$ estimation that is unbiased at the 0.1 per cent level, and with extremely well characterized photo- $z$ errors (Fig. 10, righthand panel).

Despite all of these successes, some additional work clearly remains. First, the current photometric redshifts must be extended into $P(z)$ distributions to properly capture skewness and kurtosis where it exists, for instance near filter transitions. Perhaps more importantly, however, the current samples clearly exhibit three distinct classes of redshift outliers. We have been able to identify the physical origin of these outliers - Clumps 1 and 3 in Fig. 3 are ellipticals or S0 galaxies with residual star formation, while Clump 2 galaxies are very dusty $(E(B-V) \approx 0.15)$ elliptical/S0 galaxies. These dusty galaxies also exhibit residual star formation, but the primary reason they are outliers is their high dust content. We defer the question of whether it is possible to photometrically identify these outliers and remove them from the redMaGiC sample to future work.

\section{ACKNOWLEDGEMENTS}

This paper has gone through internal review by the DES collaboration. We are grateful for the extraordinary contributions of our CTIO colleagues and the DECam Construction, Commissioning and Science Verification teams in achieving the excellent instrument and telescope conditions that have made this work possible. The success of this project also relies critically on the expertise and dedication of the DES Data Management group.

This work was supported in part by the US Department of Energy contract to SLAC no. DE-AC02-76SF00515.

Funding for the DES Projects has been provided by the US Department of Energy, the US National Science Foundation, the Ministry of Science and Education of Spain, the Science and Technology Facilities Council of the United Kingdom, the Higher Education Funding Council for England, the National Center for Supercomputing Applications at the University of Illinois at Urbana-Champaign, the Kavli Institute of Cosmological Physics at the University of Chicago, the Center for Cosmology and Astro-Particle Physics at the Ohio State University, the Mitchell Institute for Fundamental Physics and Astronomy at Texas A\&M University, Financiadora de Estudos e Projetos, Fundação Carlos Chagas Filho de Amparo à Pesquisa do Estado do Rio de Janeiro, Conselho Nacional de Desenvolvimento Científico e Tecnológico and the Ministério da Ciência, Tecnologia e Inovação, the Deutsche Forschungsgemeinschaft, and the Collaborating Institutions in the Dark Energy Survey. The DES data management system is supported by the National Science Foundation under Grant Number AST-1138766.

The Collaborating Institutions are Argonne National Laboratory, the University of California at Santa Cruz, the University of Cambridge, Centro de Investigaciones Enérgeticas, Medioambientales y Tecnológicas-Madrid, the University of Chicago, University College London, the DES-Brazil Consortium, the University of Edinburgh, the Eidgenössische Technische Hochschule (ETH) Zürich, Fermi National Accelerator Laboratory, the University of Illinois at Urbana-Champaign, the Institut de Ciències de 1'Espai (IEEC/CSIC), the Institut de Física d'Altes Energies, Lawrence Berkeley National Laboratory, the Ludwig-Maximilians Universität München and the associated Excellence Cluster Universe, the University of Michigan, the National Optical Astronomy Observatory, the University of Nottingham, the Ohio State University, the University of Pennsylvania, the University of Portsmouth, SLAC National Accelerator Laboratory, Stanford University, the University of Sussex, and Texas A\&M University.

The DES participants from Spanish institutions are partially supported by MINECO under grants AYA2012-39559, ESP201348274, FPA2013-47986, and Centro de Excelencia Severo Ochoa SEV-2012-0234. Research leading to these results has received funding from the European Research Council under the European Union's Seventh Framework Programme (FP7/2007-2013) including ERC grant agreements 240672, 291329, and 306478.

This work is based in part on observations taken at the Australian Astronomical Observatory under programme A/2013B/012.

Funding for SDSS-III has been provided by the Alfred P. Sloan Foundation, the Participating Institutions, the National Science Foundation, and the US Department of Energy Office of Science. The SDSS-III website is http://www.sdss3.org/.

SDSS-III is managed by the Astrophysical Research Consortium for the Participating Institutions of the SDSS-III Collaboration including the University of Arizona, the Brazilian Participation Group, Brookhaven National Laboratory, University of Cambridge, Carnegie Mellon University, University of Florida, the French Participation Group, the German Participation Group, 
Harvard University, the Instituto de Astrofisica de Canarias, the Michigan State/Notre Dame/JINA Participation Group, Johns Hopkins University, Lawrence Berkeley National Laboratory, Max Planck Institute for Astrophysics, Max Planck Institute for Extraterrestrial Physics, New Mexico State University, New York University, Ohio State University, Pennsylvania State University, University of Portsmouth, Princeton University, the Spanish Participation Group, University of Tokyo, University of Utah, Vanderbilt University, University of Virginia, University of Washington, and Yale University.

This publication makes use of data products from the Two Micron All Sky Survey, which is a joint project of the University of Massachusetts and the Infrared Processing and Analysis Center/California Institute of Technology, funded by the National Aeronautics and Space Administration and the National Science Foundation.

GAMA is a joint European-Australasian project based around a spectroscopic campaign using the Anglo-Australian Telescope. The GAMA input catalogue is based on data taken from the Sloan Digital Sky Survey and the UKIRT Infrared Deep Sky Survey. Complementary imaging of the GAMA regions is being obtained by a number of independent survey programmes including GALEX MIS, VST KiDS, VISTA VIKING, WISE, Herschel-ATLAS, GMRT, and ASKAP providing UV-to-radio coverage. GAMA is funded by the STFC (UK), the ARC (Australia), the AAO, and the participating institutions. The GAMA website is http://www.gama-survey.org/.

\section{REFERENCES}

Abazajian K. N. et al., 2009, ApJS, 182, 543

Abdalla F. B., Banerji M., Lahav O., Rashkov V., 2011, MNRAS, 417, 1891

Ahn C. P. et al., 2014, ApJS, 211, 17

Aihara H. et al., 2011, ApJS, 193, 29

Annis J. et al., 2014, ApJ, 794, 120

Benítez N., 2000, ApJ, 536, 571

Bertin E., 2010, Astrophysics Source Code Library, record ascl:1010.068

Bertin E., 2011, in Evans I. N., Accomazzi A., Mink D. J., Rots A. H., eds, ASP Conf. Ser. Vol. 442, Astronomical Data Analysis Software and Systems XX. Astron. Soc. Pac., San Francisco, p. 435

Bertin E., Arnouts S., 1996, A\&AS, 117, 393

Blanton M. R., Roweis S., 2007, AJ, 133, 734

Bleem L. E. et al., 2015, ApJS, 216, 27

Bonnett C., 2015, MNRAS, 449, 1043

Bonnett C. et al., 2015, preprint (arXiv:1507.05909)

Bradshaw E. J. et al., 2013, MNRAS, 433, 194

Bruzual G., Charlot S., 2003, MNRAS, 344, 1000

Budavári T., Szalay A. S., Connolly A. J., Csabai I., Dickinson M., 2000, AJ, 120, 1588

Carlstrom J. E. et al., 2011, PASP, 123, 568

Colless M., Dalton G., Maddox S., Sutherland W., the $2 \mathrm{dF}$ collaboration, 2001, MNRAS, 328, 1039

Collister A. A., Lahav O., 2004, PASP, 116, 345

Cooper M. C. et al., 2012, MNRAS, 425, 2116

Csabai I., Dobos L., Trencséni M., Herczegh G., Józsa P., Purger N., Budavári T., Szalay A. S., 2007, Astron. Nachr., 328, 852

Dawson K. S. et al., 2013, AJ, 145, 10

Desai S. et al., 2012, ApJ, 757, 83

Diehl T. et al., 2012, Phys. Procedia, 37, 1332

Driver S. P. et al., 2011, MNRAS, 413, 971

Eisenstein D. J. et al., 2001, AJ, 122, 2267

Eisenstein D. J. et al., 2005, ApJ, 633, 560

Flaugher B. et al., 2015, AJ, 150, 150

Franzetti P., Garilli B., Guzzo L., Marchetti A., Scodeggio M., 2014, preprint (arXiv:e-prints)

Garilli B. et al., 2008, A\&A, 486, 683
Garilli B. et al., 2014, A\&A, 562, A23

Górski K. M., Hivon E., Banday A. J., Wandelt B. D., Hansen F. K., Reinecke M., Bartelmann M., 2005, ApJ, 622, 759

Graff P., Feroz F., Hobson M. P., Lasenby A., 2014, MNRAS, 441, 1741

Hoffleit D., Jaschek C., 1991, The Bright Star Catalogue

Hoyle B., Rau M. M., Zitlau R., Seitz S., Weller J., 2015a, MNRAS, 449, 1275

Hoyle B., Rau M. M., Bonnett C., Seitz S., Weller J., 2015b, MNRAS, 450, 305

Jarvis M., Bernstein G., Jain B., 2004, MNRAS, 352, 338

Jarvis M. et al., 2016, MNRAS, 460, 2245

Kelly P. L. et al., 2014, MNRAS, 439, 28

LSST Science Collaboration 2009, preprint (arXiv:0912.0201)

McLure R. J. et al., 2013, MNRAS, 428, 1088

Nelder J. A., Mead R., 1965, Comput. J., 308

O’Donnell J. E., 1994, ApJ, 422, 158

Ross A. J. et al., 2011, MNRAS, 417, 1350

Rozo E., Rykoff E. S., Becker M., Reddick R. M., Wechsler R. H., 2015, MNRAS, 453, 38

Rykoff E. S. et al., 2014, ApJ, 785, 104 (RM1)

Rykoff E. S. et al., 2016, ApJS, 224, 1

Sánchez C. et al., 2014, MNRAS, 445, 1482

Sevilla I. et al., 2011, preprint (arXiv:1109.6741)

Sinnott R. W., 1988, The Complete Nnew General Catalogue and Index Catalogues of Nebulae and Star Clusters by J. L. E. Dreyer

Skrutskie M. F. et al., 2006, AJ, 131, 1163

Stoughton C. et al., 2002, AJ, 123, 485

The Dark Energy Survey Collaboration 2005, preprint (arXiv:e-prints)

Tojeiro R. et al., 2014, MNRAS, 440, 2222

York D. G. et al., 2000, AJ, 120, 1579

Yuan F. et al., 2015, MNRAS, 452, 3047

\section{APPENDIX A: STAR-GALAXY SEPARATION}

To perform star/galaxy separation, we use object size estimates from the NGMIX multi-epoch shape fitting catalogue (Jarvis et al. 2016). The NGMIX algorithm fits an exponential disc profile to each object (in all individual observations of each griz band), and estimates an intrinsic (PSF-deconvolved) size (exp_t), as well as an error on that size (exp_t_err). Fig. A1 shows a distribution of object sizes as a function of magnitude in the SPTE footprint. The stellar locus at zero-size is obviously separated from the galaxy locus at the bright

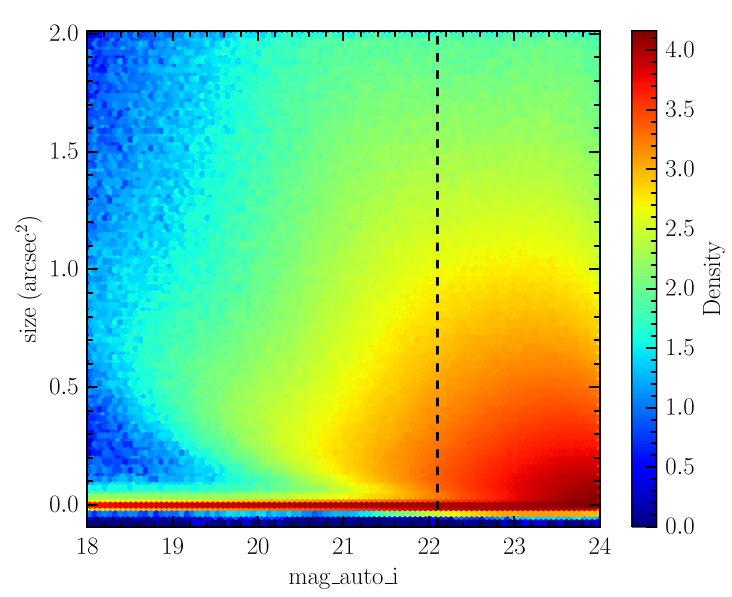

Figure A1. Intrinsic object size, exp_t $t$, as a function of $m_{i}$ (as estimated with MAG_AUTO). At the bright end, the stars are clearly separated from the galaxies, while the confusion is apparent at $m_{i} \sim 23$. The magnitude of the galaxies in the redMaGiC sample described here, with $z \lesssim 0.8$ and $L / L_{*}>0.5$, is shown with a dashed black line. 
end. At the faint end, where the intrinsic size of the galaxies is close to the typical seeing, it is harder to distinguish between the two loci. Our goal here is to select as complete a galaxy sample as possible while minimizing stellar contamination. Our task is made a little easier by the fact that we are limiting ourselves to red galaxies with $z \leq 0.8$ and $L / L_{*}>0.5$, the magnitude limit of which is denoted with a dashed red line in the figure.

Until we develop a full probabilistic star/galaxy separator from the NGMIX size estimator, we have decided to make use of simple cuts based on the intrinsic size and error on the size. At the bright end, we see that true stars do not have intrinsic size exp_t $>0.002$. At the faint end, we wish to make a selection that has as high a galaxy completeness as possible, minimizing stellar contamination. We make the ansatz that such a cut will take the form

exp_t $+n \times$ exp_t_err $>0.002$,

where $n$ is some number to be determined, and we expect $n \approx 2$. That is, we keep all objects that are consistent with being extended sources within observational errors.

In order to choose a value of $n$, we have decided to make use of cross-correlation tests. Specifically, stars and galaxies should be uncorrelated with each other. Consequently, a non-zero crosscorrelation between a galaxy sample and a known stellar sample is indicative of stellar contamination in the galaxy sample.

Consider a sample of $n$ total objects that contains $n_{\mathrm{g}}$ galaxies and $n_{*}$ stars. One has then

$n=\bar{n}_{\mathrm{g}}\left(1+\delta_{\mathrm{g}}\right)+\bar{n}_{*}\left(1+\delta_{*}\right)$,

and therefore

$1+\delta=\frac{\bar{n}_{\mathrm{g}}}{\bar{n}}\left(1+\delta_{\mathrm{g}}\right)+\frac{\bar{n}_{*}}{\bar{n}}\left(1+\delta_{*}\right)$.

Defining the stellar fraction of the sample $f_{*}=\bar{n}_{*} / \bar{n}_{\mathrm{g}}$, we arrive at

$\delta=\left(1-f_{*}\right) \delta_{\mathrm{g}}+f_{*} \delta_{*}$.

Now, if we cross-correlate this sample (subscript 'obs') with a known sample of stars, then we have

$w_{\mathrm{obs}, \mathrm{s}}=\left\langle\delta_{\mathrm{s}} \delta\right\rangle=f_{*}\left\langle\delta_{\mathrm{s}} \delta_{*}\right\rangle=f_{*}\left\langle\delta_{\mathrm{s}} \delta_{\mathrm{s}}\right\rangle=f_{*} w_{\mathrm{s}, \mathrm{s}}$,

where $\delta_{\mathrm{s}}$ is the fluctuation of a known stellar population, and we have assumed $\delta_{\mathrm{s}}=\delta_{*}$. It follows from this assumption that the crosscorrelation $w_{\mathrm{obs}, \mathrm{s}}$ is proportional to the stellar auto-correlation $w_{\mathrm{s}, \mathrm{s}}$. Consequently, we can estimate the stellar contamination via

$f_{*}=\frac{w_{\mathrm{s}, \mathrm{s}}}{w_{\mathrm{obs}, \mathrm{s}}}$.

By computing the above ratio for a galaxy selected using a cut $n$ as per equation (A1), we seek to optimize our sample selection. To measure the cross-correlations, we make use of the TREECORR code (Jarvis, Bernstein \& Jain 2004). $f_{*}$ is obtained by computing the median value of the above ratio on scales of 1-10 arcmin.

We can use a similar method to estimate the completeness associated with our stellar-galaxy separation cut. Specifically, consider again equation (A1). For large $n$, the selected sample should be highly complete. Suppose that at a large $n$, call it $n_{\max }$, the sample has $N\left(n_{\max }\right)$ objects, and a stellar fraction $f_{*}\left(n_{\max }\right)$ estimated via cross-correlations. It follows that the number of galaxies is $N\left(n_{\max }\right) f_{*}\left(n_{\max }\right)$. At a lower $n$, the number of galaxies $N(n) f_{*}(n)$ will have decreased, and the relative completeness is simply

$C(n)=\frac{N(n) f_{*}(n)}{N\left(n_{\max }\right) f_{*}\left(n_{\max }\right)}$.

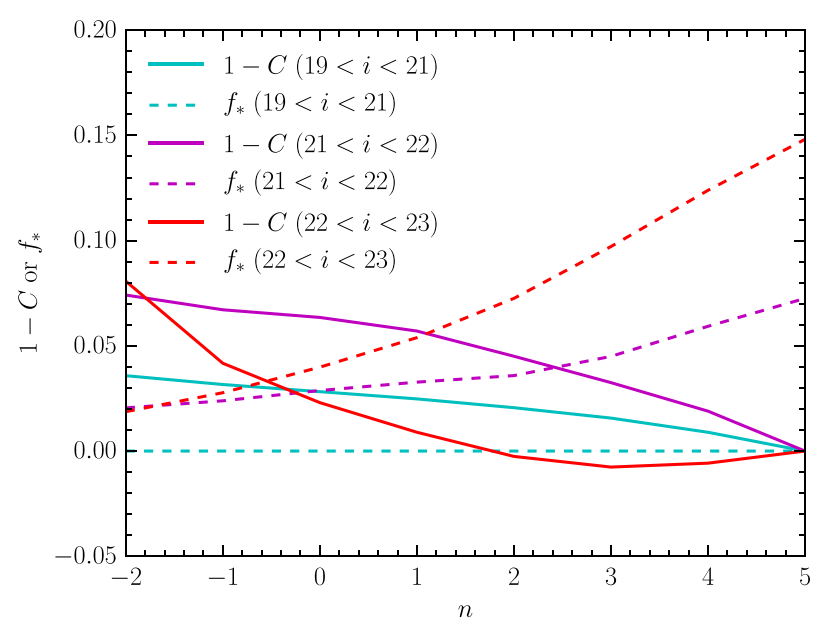

Figure A2. Incompleteness $(1-C$, dashed lines) and stellar contamination ( $f_{*}$, solid lines) for four different magnitude bins, as a function of the selection parameter $n$. The fainter galaxies tend to have lower completeness and larger stellar contamination.

We set $n_{\max }=5$ to define the relative completeness, and look for the value of $n$ which results in the best compromise between purity and completeness.

We have implemented the above method with two stellar selections, a bright sample $(19.0<i<21.5)$ and a faint sample $(21.5$ $<i<22.5$ ). Fig. A2 shows the results for the faint sample. Results for the bright sample are difficult to interpret (see below for further details). The solid lines in Fig. A2 show the incompleteness $(1-C)$ as a function of $n$ for three different magnitude bins. The dashed lines show the $f_{*}$ value for the same bins. The faintest galaxies in the fiducial redMaGiC sample have $i \approx 22$, and thus lie in between the red and purple lines. The point $f_{*}=1-C$ for these two lines is $n \approx-0.5$ and $n \approx 2.5$, respectively. We adopt as our fiducial cut the mean of the these two values, $n=1$. From the figure, we expect $\approx 4$ per cent stellar contamination and 4 per cent galaxy incompleteness.

Results from the bright stellar reference sample are difficult if not impossible to interpret. For instance, the completeness $C$ estimated as above using the bright sample is larger than unity. The estimated stellar fraction using the bright stellar reference sample is $\approx 10$ per cent. The difference between the bright and faint stellar reference samples suggests that the assumption $\delta_{\mathrm{s}}=\delta_{*}$ is in fact incorrect, and that a more reasonable model might be $\delta_{\mathrm{s}}=k \delta_{*}$ for some $k$. Since all we seek here is an optimal star-galaxy separation criterion, we adopt the proposed cut with $n=1$ here, and leave the problem of a more accurate estimate of the stellar contamination for the redMaGiC galaxy sample to future work.

We emphasize that the stellar contamination fractions quoted above are those relevant for the full galaxy catalogue given the stargalaxy separation criterion we have adopted. The stellar fraction of the redMaGiC catalogue is much suppressed, since an object must also have red-sequence colours in order to make it into the redMaGiC catalogue. The only redshift at which the stellar locus crosses the red sequence is $z \approx 0.7$, so we expect $\approx 5$ per cent stellar contamination at $z \approx 0.7$, but essentially no contamination at other redshifts.

\section{APPENDIX B: DATA CATALOGUE FORMATS}

The full redMaGiC SDSS DR8 and DES SV catalogues will be available at http://risa.stanford.edu/redmapper/ in FITS format, and 
Table B1. redMaGiC SDSS DR8 redMaGiC catalogue format.

\begin{tabular}{|c|c|c|c|}
\hline Column & Name & Format & Description \\
\hline 1 & OBJID & $\mathrm{I} 18$ & SDSS DR8 CAS object identifier \\
\hline 2 & RA & F12.7 & Right ascension in decimal degrees (J2000) \\
\hline 3 & $\mathrm{DEC}$ & F12.7 & Declination in decimal degrees (J2000) \\
\hline 4 & IMAG & F6.3 & SDSS $i$ CMODEL magnitude (dereddened) \\
\hline 5 & IMAG_ERR & F6.3 & Error on $i$ CMODEL magnitude \\
\hline 6 & MODEL_MAG_U & F6.3 & SDSS $u$ model magnitude (dereddened) \\
\hline 7 & MODEL_MAGERR_U & F6.3 & Error on $u$ model magnitude \\
\hline 8 & MODEL_MAG_R & F6.3 & SDSS $g$ model magnitude (dereddened) \\
\hline 9 & MODEL_MAGERR_R & F6.3 & Error on $g$ model magnitude \\
\hline 10 & MODEL_MAG_I & F6.3 & SDSS $r$ model magnitude (dereddened) \\
\hline 11 & MODEL_MAGERR_I & F6.3 & Error on $r$ model magnitude \\
\hline 12 & MODEL_MAG_Z & F6.3 & SDSS $i$ model magnitude (dereddened) \\
\hline 13 & MODEL_MAGERR_Z & F6.3 & Error on $i$ model magnitude \\
\hline 14 & MODEL_MAG_Y & F6.3 & SDSS $z$ model magnitude (dereddened) \\
\hline 15 & MODEL_MAGERR_Y & F6.3 & Error on $z$ model magnitude \\
\hline 16 & MABS_U & F6.3 & Absolute magnitude in $u$ \\
\hline 17 & MABS_ERR_U & F6.3 & Error on absolute magnitude in $u$ \\
\hline 18 & MABS_G & F6.3 & Absolute magnitude in $g$ \\
\hline 19 & MABS_ERR_G & F6.3 & Error on absolute magnitude in $g$ \\
\hline 20 & MABS_R & F6.3 & Absolute magnitude in $r$ \\
\hline 21 & MABS_ERR_R & F6.3 & Error on absolute magnitude in $r$ \\
\hline 22 & MABS_I & F6.3 & Absolute magnitude in $i$ \\
\hline 23 & MABS_ERR_I & F6.3 & Error on absolute magnitude in $i$ \\
\hline 24 & MABS_Z & F6.3 & Absolute magnitude in $z$ \\
\hline 25 & MABS_ERR_Z & F6.3 & Error on absolute magnitude in $z$ \\
\hline 26 & ILUM & F6.3 & $i$-band luminosity, units of $L_{*}$ \\
\hline 26 & ZREDMAGIC & F6.3 & redMaGiC photometric redshift \\
\hline 27 & ZREDMAGIC_E & F6.3 & Error on redMaGiC photometric redshift \\
\hline 28 & CHISQ & F6.3 & $\chi^{2}$ of fit to redMaGiC template \\
\hline 29 & Z_SPEC & F8.5 & SDSS spectroscopic redshift ( -1.0 if not available) \\
\hline
\end{tabular}

Table B2. redMaGiC DES SV redMaGiC catalogue format.

\begin{tabular}{lccc}
\hline Column & Name & Format & Description \\
\hline 1 & COADD_OBJECT_ID & I18 & DES SVA1 object identifier \\
2 & RA & F12.7 & Right ascension in decimal degrees (J2000) \\
3 & DEC & F12.7 & Declination in decimal degrees (J2000) \\
4 & MAG_AUTO_G & F6.3 & $g$ MAG_AUTO magnitude (SLR corrected) \\
5 & MAGERR_AUTO_G & F6.3 & Error on $g$ MAG_AUTO magnitude \\
6 & MAG_AUTO_R & F6.3 & $r$ MAG_AUTO magnitude (SLR corrected) \\
7 & MAGERR_AUTO_R & F6.3 & Error on $r$ MAG_AUTO magnitude \\
8 & MAG_AUTO_I & F6.3 & $i$ MAG_AUTO magnitude (SLR corrected) \\
9 & MAGERR_AUTO_I & F6.3 & Error on $i$ MAG_AUTO magnitude \\
10 & MAG_AUTO_Z & F6.3 & $z$ MAG_AUTO magnitude (SLR corrected) \\
11 & MAGERR_AUTO_Z & F6.3 & Error on $z$ MAG_AUTO magnitude \\
12 & MABS_G & F6.3 & Absolute magnitude in $g$ \\
13 & MABS_ERR_G & F6.3 & Error on absolute magnitude in $g$ \\
14 & MABS_R & F6.3 & Absolute magnitude in $r$ \\
15 & MABS_ERR_R & F6.3 & Error on absolute magnitude in $r$ \\
16 & MABS_I & F6.3 & Absolute magnitude in $i$ \\
17 & MABS_ERR_I & F6.3 & Error on absolute magnitude in $i$ \\
18 & MABS_Z & F6.3 & Absolute magnitude in $z$ \\
19 & MABS_ERR_Z & F6.3 & Error on absolute magnitude in $z$ \\
20 & ZLUM & F6.3 & $z$-band luminosity, units of $L *$ \\
21 & ZREDMAGIC & F6.3 & redMaGiC photometric redshift \\
22 & ZREDMAGIC_E & F6.3 & Error on redMaGiC photometric redshift \\
23 & CHISQ & F6.3 & $\chi$ of fit to redMaGiC template \\
24 & Z_SPEC & F8.5 & Spectroscopic redshift $(-1.0$ if not available) \\
\hline & & &
\end{tabular}


from the online journal in machine-readable formats. A summary of the DR8 catalogue is given in Table B1, and the SV catalogue is given in Table B2. Absolute magnitudes in the tables are computed using KCORRECT v4.2 (Blanton \& Roweis 2007). $k$-corrections are applied assuming an LRG template, band shifted to $z=0.1$.

The SDSS catalogues will be made publicly available upon publication of this paper in a journal. We plan to release the DES redMaGiC catalogues publicly by 2016 January. See the Dark Energy Survey website ${ }^{3}$ for instructions on how to download the catalogues.

${ }^{1}$ Department of Physics, University of Arizona, Tucson, AZ 85721, USA

${ }^{2}$ Kavli Institute for Particle Astrophysics \& Cosmology, PO Box 2450, Stanford University, Stanford, CA 94305, USA

${ }^{3}$ SLAC National Accelerator Laboratory, Menlo Park, CA 94025, USA

${ }^{4}$ Institut de Física d'Altes Energies, Universitat Autònoma de Barcelona, E-08193 Bellaterra, Barcelona, Spain

${ }^{5}$ Institut de Ciències de l'Espai, IEEC-CSIC, Campus UAB, Carrer de Can Magrans, s/n, E-08193 Bellaterra, Barcelona, Spain

${ }^{6}$ ARC Centre of Excellence for All-sky Astrophysics (CAASTRO), 44 Rosehill Street, Redfern, NSW 2016, Australia

${ }^{7}$ School of Mathematics and Physics, University of Queensland, QLD 4072, Australia

${ }^{8}$ Universitäts-Sternwarte, Fakultät für Physik, Ludwig-Maximilians Universität München, Scheinerstr. 1, D-81679 München, Germany

${ }^{9}$ Department of Physics \& Astronomy, University College London, Gower Street, London, WC1E 6BT, UK

${ }^{10}$ Department of Physics, Stanford University, 382 Via Pueblo Mall, Stanford, CA 94305, USA

${ }^{11}$ Cerro Tololo Inter-American Observatory, National Optical Astronomy Observatory, Casilla 603, La Serena, Chile

${ }^{12}$ Institute of Astronomy, University of Cambridge, Madingley Road, Cambridge $C B 3$ OHA, UK

${ }^{13}$ Kavli Institute for Cosmology, University of Cambridge, Madingley Road, Cambridge CB3 OHA, UK

${ }^{14}$ Department of Physics and Astronomy, University of Pennsylvania, Philadelphia, PA 19104, USA

${ }^{15}$ CNRS, UMR 7095, Institut d'Astrophysique de Paris, F-75014 Paris, France

${ }^{16}$ Sorbonne Universités, UPMC Univ Paris 06, UMR 7095, Institut d'Astrophysique de Paris, F-75014 Paris, France

${ }^{17}$ Fermi National Accelerator Laboratory, PO Box 500, Batavia, IL 60510, USA

${ }^{18}$ Institute of Cosmology \& Gravitation, University of Portsmouth, Portsmouth PO1 3FX, UK

${ }^{19}$ Laboratório Interinstitucional de e-Astronomia - LIneA, Rua Gal. José Cristino 77, Rio de Janeiro, RJ 20921-400, Brazil

${ }^{20}$ Observatório Nacional, Rua Gal. José Cristino 77, Rio de Janeiro, RJ 20921-400, Brazil

${ }^{21}$ University of Notre Dame, Notre Dame, IN 46556, USA
${ }^{22}$ Department of Astronomy, University of Illinois, 1002 W. Green Street, Urbana, IL 61801, USA

${ }^{23}$ National Center for Supercomputing Applications, 1205 West Clark St., Urbana, IL 61801, USA

${ }^{24}$ Research School of Astronomy and Astrophysics, Australian National University, Canberra, ACT 2611, Australia

${ }^{25}$ George P. and Cynthia Woods Mitchell Institute for Fundamental Physics and Astronomy, and Department of Physics and Astronomy, Texas A\&M University, College Station, TX 77843, USA

${ }^{26}$ Excellence Cluster Universe, Boltzmannstr. 2, D-85748 Garching, Germany

${ }^{27}$ Faculty of Physics, Ludwig-Maximilians University, Scheinerstr. 1, D81679 Munich, Germany

${ }^{28}$ Jet Propulsion Laboratory, California Institute of Technology, 4800 Oak Grove Dr., Pasadena, CA 91109, USA

${ }^{29}$ Department of Astronomy, University of Michigan, Ann Arbor, MI 48109, USA

${ }^{30}$ Department of Physics, University of Michigan, Ann Arbor, MI 48109, USA

${ }^{31}$ Kavli Institute for Cosmological Physics, University of Chicago, Chicago, IL 60637, USA

${ }^{32}$ Centre for Astrophysics \& Supercomputing, Swinburne University of Technology, VIC 3122, Australia

${ }^{33}$ Max Planck Institute for Extraterrestrial Physics, Giessenbachstrasse, D-85748 Garching, Germany

${ }^{34}$ Center for Cosmology and Astro-Particle Physics, The Ohio State University, Columbus, $\mathrm{OH} 43210$, USA

${ }^{35}$ Department of Physics, The Ohio State University, Columbus, OH 43210, USA

${ }^{36}$ Lawrence Berkeley National Laboratory, 1 Cyclotron Road, Berkeley, CA 94720, USA

${ }^{37}$ Australian Astronomical Observatory, North Ryde, NSW 2113, Australia

${ }^{38}$ Departamento de Física Matemática, Instituto de Física, Universidade de São Paulo, CP 66318, CEP 05314-970, São Paulo, SP, Brazil

${ }^{39}$ Department of Astronomy, The Ohio State University, Columbus, OH 43210, USA

${ }^{40}$ Institució Catalana de Recerca i Estudis Avançats, E-08010 Barcelona, Spain

${ }^{41}$ Department of Physics and Astronomy, Pevensey Building, University of Sussex, Brighton BN1 9QH, UK

${ }^{42}$ Centro de Investigaciones Energéticas, Medioambientales y Tecnológicas (CIEMAT), Complutense 40, Madrid, Spain

${ }^{43}$ Instituto de Física, UFRGS, Caixa Postal 15051, Porto Alegre, RS 91501 970, Brazil

${ }^{44}$ Department of Physics, University of Illinois, 1110 W. Green St., Urbana, IL 61801, USA

${ }^{45}$ Argonne National Laboratory, 9700 South Cass Avenue, Lemont, IL 60439, USA

This paper has been typeset from a $\mathrm{T}_{\mathrm{E}} \mathrm{X} / \mathrm{L} \mathrm{AT} \mathrm{E} \mathrm{X}$ file prepared by the author.

\footnotetext{
${ }^{3}$ http://www.darkenergysurvey.org/
} 